\title{
Taxonomic Revision of the Genus Pistacia L. (Anacardiaceae)
}

\author{
Mohannad G. AL-Saghir ${ }^{1 *}$, Duncan M. Porter $^{2}$ \\ ${ }^{1}$ Department of Environmental and Plant Biology, Ohio University Zanesville, Zanesville, USA; ${ }^{2}$ Department of Biological Sciences, \\ Virginia Polytechnic Institute and State University, Blacksburg, USA. \\ Email: *al-saghi@ohio.edu
}

Received October $10^{\text {th }}, 2011$; revised November $9^{\text {th }}, 2011$; accepted November $29^{\text {th }}, 2011$

\begin{abstract}
Pistacia is an economically important genus because it contains the pistachio crop, $P$. vera, which has edible seeds of considerable commercial importance whose value has increased over the last two decades reaching an annual value of about $\$ 2$ billion (harvested crop). The taxonomic relationships among its species are controversial and not well understood due to the fact that they have no genetic barriers. The taxonomy of this genus is revised in detail through our research. It includes the following taxa: Pistacia atlantica Desf., P. chinensis Bunge subsp. chinensis, P. chinensis subsp. falcata (Bess. ex Martinelli) Rech. f., P. chinensis subsp. integerrima (J.L. Stew. ex Brandis) Rech. f., P. eurycarpa Yalt., P. khinjuk Stocks, P. lentiscus L. subsp. lentiscus, P. lentiscus subsp. emarginata (Engl.) AL-Saghir, P. mexicana Humb., Bonpl., \& Kunth, P. X saportae Burnat, P. terebinthus L., P. vera L., and P. weinmannifolia Poiss. ex Franch. The genus is divided into two sections: section Pistacia and section Lentiscella. A key to the 14 taxa that have been recognized by this study is included. The new combination P. lentiscus subsp. emarginata (Engl.) AL-Saghir is made, and the names P. chinensis subsp. integerrima (J. L. Stew. ex Brandis) Rech. F., P. terebinthus L., and P. vera L. are lectotypified.
\end{abstract}

Keywords: Taxonomy; Phylogeny; Pistacia; Anacardiaceae; Genus; Pistacia vera

\section{Introduction}

Pistacia L. belongs to the family Anacardiaceae (cashew family), order Sapindales [1]. It contains nine species and five subspecies according to the current study.

Pistacia vera L. (cultivated pistachio) is by far the most economically important species in the genus. The value of $P$. vera fruits has increased over the last two decades reaching an annual value of about $\$ 2$ billion (harvested crop) [2]. It has edible seeds and considerable commercial importance. Pistachios are utilized mostly in the shell, for fresh consumption; processed uses include candy, baked goods, and ice cream. They also have traditional, medicinal, and non-food uses such as toothache relief. The resin is used as a blood-clotting agent in Europe and the Middle East, while husks are used in India for dying and tanning. Pistachios have been reported as a remedy for scirrhus and sclerosis of the liver, abscesses, poor circulation, and other medical problems [3]. Currently, Iran, the United States, Turkey, and Syria are the main pistachio producers in the world, contributing over $90 \%$ of the world production [2].

Members of the genus are dioecious trees or shrubs

${ }^{*}$ Corresponding author. with well-developed vertical resin canals. Leaves are alternate, deciduous or persistent, pinnately compound, but sometimes trifoliolate or unifoliolate, pari-or imparipinnate, membranaceous or leathery. Inflorescences are determinate and terminal or axillary. Flowers are almost always unisexual, radial, small, with well developed staminodes or carpellodes, and apetalous; sepals are usually 5 , distinct to slightly connate; stamens are $4-5$, filaments are very short, usually glabrous, and usually distinct; pollen grains tricolporate or triporate; carpels are usually 3 and variously connate; ovary superior, with only 1 fertile and well developed carpel; gynoecium asymmetrical and unilocular with apical placentation, ovule 1; stigma capitate. Nectariferous disk present, usually intrastaminal. Fruit a flattened asymmetrical drupe, embryo curved; endosperm scanty or lacking.

Pistacia is believed to have originated in Central Asia [4-7]. Two centers of diversity have been described. One comprises the Mediterranean region of Southern Europe, Northern Africa, and the Middle East. The second comprises West and Central Asia [6-9]. The species of the genus occur naturally from North Africa to the Philippines and from Texas to Nicaragura. 
Few systematic studies have been published on this important genus. The first complete classification of the genus was published by Zohary (1952) [10]. Today, there are many questions about Zohary's taxonomic treatment, the status of many of his species, and the accuracy of dividing the genus into four sections. This disagreement is mainly because of little information being available on the phylogeny of the genus. Studies such as the one performed by Zohary (1952) were hampered by the small sampling size, weak representation of the species, and unefficient methods. Consequently, the studies failed to resolve the relationships between species within the genus. Moreover, species of Pistacia easily form interspecific hybrids, suggesting close relationships, which limit the previous studies and make the actual level of speciation and relationships within the genus unclear [4]. A better understanding of these relationships is also needed to make the species more useful for plant improvement or genetic studies.

A comprehensive taxonomic revision, utilizing different types of data, is urgently needed for Pistacia in order to clarify the phylogenetic relationships between the species and to characterize the collective germplasm. This will provide a framework and guidelines for plant improvement and help to preserve the genetic resources of this important crop, especially since these genetic resources are under threat by extensive forest cutting in their native countries (especially in the Mediterranean countries, and Central and Western Asia).

\section{Taxonomic History}

Linnaeus was the first to establish the genus. In his Species Plantarum, Linnaeus (1753) [11] recognized six species of Pistacia: P. lentiscus, P. narbonensis, P. simaruba, $P$. terebinthus, $P$. trifolia, and P. vera. Pistacia narbonensis and $P$. trifolia are synonyms of $P$. vera; $P$. simaruba is Bursera simaruba (L.) Sarg. of the closely related Burseraceae.

Prior to Linnaeus, Tournefort (1700) [12] considered $P$. lentiscus a distinct genus, Lentiscus, while he classified $P$. terebinthus and $P$. vera under the genus Terebinthus.

At present, the most complete taxonomic study is that of Zohary (1952), who included 11 species in the genus and divided them into four sections: Lentiscella Zohary (containing $P$. mexicana Humb., Bonpl. \& Kunth and $P$. texana Swingle); Eu Lentiscus Zohary (containing $P$. lentiscus L., P. saportae Burnat, and P. weinmannifolia Poiss. ex Franch.); Butmela Zohary (containing P. atlantica Desf.); and Eu Terebinthus Zohary (containing $P$. chinensis Bunge, P. khinjuk Stocks., P. palaestina Boiss., $P$. terebinthus L., and $P$. vera L.).

Since Zohary's (1952) treatment, several new species have been added to Pistacia. Yaltirik (1967a, 1967b) [13,
14] classified Pistacia species in Turkey and described a new species, $P$. eurycarpa. This had been called P. atlantica var. kurdica by Zohary (1952), a synonym. Zohary considered $P$. palaestina as a separate species, whereas Yaltirik (1967a, 1967b) retained it as a variety of P. terebinthus. Kokwaro described a new species from East Africa, P. aethiopica, in Kokwaro and Gillett (1980).

Fifty-five binomials for Pistacia were found in the International Plant Names Index [15], only 14 were recognized by the authors (all those listed in this section). The hybrids will be further discussed in the taxonomic section of this study.

Yi et al. (2008) [16] assessed the phylogeny of Pistacia using five molecular sets, sequences of nuclear ribosomal ITS, the third intron of the nuclear nitrate reductase gene (NIA-i3), and the plastid $n d h F, \operatorname{trnL}-F$, and $\operatorname{trnC}$-trnD. Their molecular data were largely consistent with our independently derived intrageneric classification based on morphology. They recognized the following species: P. atlantica, $P$. chinensis, $P$. integerrima, $P$. khinjuk, P. lentiscus, P. mexicana, P. palaestina, $P$. saportae, $P$. terebinthus, $P$. texana, $P$. vera, and $P$. weinmannifolia. Except for $P$. palaestina and $P$. texana, our study recognized these taxa $(P$. integerrima as a subspecies of $P$. chinensis), plus $P$. chinensis subsp. falcata, $P$. eurycarpa, and $P$. lentiscus subsp. emarginata. Pistacia was shown to be monophyletic in all analyses. The two accessions of $P$. vera formed a clade with $P$. khinjuk in all molecular data sets. Some of the ITS and NIA-i3 sequences of these two species were identical, suggesting a close relationship. Earlier molecular results also suggested a close relationship between them [4-6, 17]. Pistacia palaestina was not well separated from $P$. terebinthus in either the plastid or the nuclear DNA data sets, and Yi et al. (2008, p. 245) stated that, "Pistacia palaestina may need to be merged into $P$. terebinthus." Close relationships between these two species were also suggested by the AFLP and the RAPD results [17-19]. These results are consistent with Engler (1936) [20] and Yaltirik (1967a), along with our classification, in considering $P$. palaestina to be a synonym of $P$. terebinthus. Pistacia mexicana and P. texana were not distinguishable in plastid restriction analyses [4]. The ITS data suggest that $P$. mexicana and $P$. texana are sister taxa; and the sequence divergence between these two species is low. Our morphological data indicate that there is too little variation to warrant the recognition of two species. Pistacia saportae was shown to be a hybrid between $P$. lentiscus (maternal) and P. terebinthus (paternal), as others had hypothesized (e.g., Zohary 1952).

Neighbor joining and parsimony analyses were performed on the morphological data for section Pistacia using PAUP 4.0 b10 [7,21]. Both analyses show that 
Section Pistacia contains three groups, the first group includes $P$. atlantica, $P$. mutica, and P. eurycarpa, the second includes $P$. chinensis, $P$. falcata, and $P$. integerrima and the third group contains $P$. khinjuk, $P$. palaestina, P. terebinthus, and P. vera. Neighbor joining and parsimony analyses were performed on the morphological data for section Lentiscella using PAUP 4.0 b10 [7,21]. Section Lentiscella, as indicated by the two analyses, is monophyletic

\section{The Genus Pistacia and Its Sectional Subdivision}

Pistacia L., Sp. Pl. 2:1025. 1753. Type: Pistacia vera L. Hitchcock and Green, Prop. Brit. Bot.: 191. 1929 (lectotype: not designated. Reg. Veg. 127: 77. 1993). Pistacia vera is lectotypified under its treatment below.

Terebinthus Mill., Gard. Dict. Abr. Ed. 4. 1754.

Lentiscus Mill., Gard. Dict. Abr. Ed. 4. 1754.

Evrardia Adans., Fam. Pl. 2: 342. 1763.

Members of the genus are dioecious trees or shrubs with well-developed vertical resin canals. Leaves are alternate, deciduous or persistent, pinnately compound, but sometimes trifoliolate or unifoliolate, pari-or imparipinnate, memberanous or leathery. Inflorescences are determinate and terminal or axillary. Flowers are almost always unisexual, radial, small, with well developed staminodes or carpellodes, and apetalous; sepals are usually 5 , distinct to slightly connate; stamens are $4-5$, filaments are very short, usually glabrous, and usually distinct; pollen grains tricolporate or triporate; carpels are usually 3 and variously connate; ovary superior, with only 1 fertile and well developed carpel; gynoecium asymmetrical and unilocular with apical placentation, ovule 1; stigma capitate. Nectariferous disk present, usually intrastaminal. Fruit a flattened asymmetrical drupe, embryo curved; endosperm scanty or lacking.

Common names.-Pistache, Pistachio [10].

\subsection{Key to the Species of the Pistacia}

1. Leaves large, $8-23 \mathrm{~cm}$ long, $5.2-23 \mathrm{~cm}$ wide.

2. Leaf rachis not winged.

3. Leaves coriaceous or becoming coriaceous

4. Petiole flattened; leaflets $3-5$, obtuse or mucronulate.............................................. vera

4. Petiole rounded, rarely flattened; leaflets (3-)6-11, mucronate....................... terebinthus

3. Leaves membranaceous.

5. Petiole angled or rounded; leaflets $1-9$, acuminate..................................... khinjuk

5. Petiole angled or flattened; leaflets $6-15$, attenuate.

6. Leaves imparipinnate

P. chinensis subsp. falcata
6. Leaflets paripinnate.

7. Leaflets broadly lanceolate, $6-11.5 \mathrm{~cm}$ long, 2 - $4 \mathrm{~cm}$ wide, average ratio $3.4-1$. .... P. chinensis subsp. integerrima

7. Leaflets lanceolate, $4-10 \mathrm{~cm}$ long, 9 - $24 \mathrm{~mm}$ wide, average ratio $4.1-1$

P. chinensis subsp. chinensis

2. Leaf rachis winged.

8. Leaves coriaceous, to $10 \mathrm{~cm}$ long, evergreen... .P. $\times$ saportae

8. Leaves membranaceous. $8-18.2 \mathrm{~cm}$ long, deciduous

9. Leaflets (1-3-)5-7, 4.5 - $6.5 \mathrm{~cm}$ long, 1.5 $3.8 \mathrm{~cm}$ wide, average ratio $2.3-1$

..P. eurycarpa

9. Leaflets (5-)7-11, 3 - $7 \mathrm{~cm}$ long, 5 - $20 \mathrm{~mm}$ wide, average ratio $3.5-1 \ldots \ldots \ldots . .$. . atlantica

1. Leaves small, $2-15.1 \mathrm{~cm}$ long, $1.8-10 \mathrm{~cm}$ wide.

10. Leaves coriaceous.

11. Leaflets 4 - 10, mucronate, lacking large lacking large distinct veins

................. lentiscus subsp. lentiscus

11. Leaflets 6 - 16, emarginate, with large distinct veins.... ............ lentiscus subsp. emarginata

10. Leaves membranaceous.

12. Leaves paripinnate; leaflets (4-6)12-20, often alternate.........P. weinmannifolia

12. Leaves imparipinnate; leaflets $10-30$, never alternate................ mexicana.

\subsection{Pistacia L. sect. Pistacia, Zohary, Palestine J. Bot. Jerusalem Ser. 5:194. 1952}

Eu Terebinthus Zohary, Palestine J. Bot. Jerusalem Ser. 5: 196. 1952. Terebinthus Parfitt \& Badanes, Proc. Natl. Acad. Sci. USA. 94:7991. 1997. Pistacia section Butmela is a taxonomic synonym based on P. atlantica Desf. Pistacia section Eu Terebinthus is an illegitimate name, because the epithet in the name of a subdivision of a genus is not to be formed from the name of the genus to which it belongs by adding the prefix Eu-(ICBN, Art. 21.3, [22]). Pistacia section Terebinthus was published without a Latin diagnosis. Type: Pistacia vera $\mathrm{L}$.

Deciduous small trees or shrubs; leaves pari- or imparipinnate, rarely trifoliolate or simple; leaflets $4-24$; rachis winged or wingless; drupes with bony endocarp.

Includes the following species: $P$. atlantica Desf., $P$. chinensis Bunge, $P$. eurycarpa Yaltirik, $P$. khinjuk Stocks, $P$. terebinthus L., and P. vera $\mathrm{L}$.

\subsubsection{Pistacia atlantica Desf., Fl. Atlant. 2:364. 1799} Lentiscus atlantica (Desf.) Kuntze, Revis. Gen. Pl. 152. 1891. Type: Probably Algeria, Desfontaines (BM) [10] 
(Figure 1).

Pistacia chia Desf., Tabl. Ecol. Bot. 199. 1804. TYPE: not designated.

Pistacia atlantica var. latifolia DC., Prodr. 2:61. 1825. TYPE: GREECE: Chios, 1822, Olivier (G).

Pistacia mutica Fisch. \& C.A. Mey., Bull. Soc. Imp. Naturalistes Moscau 4:338. 1838. Lentiscus mutica (Fisch. \& C.A. Mey.) Kuntze, Revis. Gen. P1. 153. 1891. Pistacia atlantica subsp. mutica (Fisch. \& C.A. Mey.) Rech. f., Fl. Iranica 63:4. 1969. TYPE: AZERBAIJAN: Goygol Rayon, Helenendorf (Goygol), Serahäzi (LE).:

Pistacia cabulica Stocks, J. Bot. Kew Gard. Misc. 4: 143. 1852. Pistacia mutica subsp. cabulica (Stocks) Engl. in A. \& C. DC., Monogr. Phan. 4:288. 1883. Pistacia atlantica subsp. cabulica (Stocks) Rech f. Fl. Iranica 63:5. 1969. TYPE: PAKISTAN: Balochistan, Stocks, 1849 (K).

Pistacia choulettei Gand., Dec. Pl. Nov. 1:44. 1875. nom. illeg. (based on P. atlantica Desf.)

Pistacia mutica f. multijuga Engl. in A. \& C. DC., Monogr. Phan. 4: 288. 1883. TYPE: ARMENIA: J.N. Szovits (W).

Pistacia atlantica var. cypricola H. Lindb., Acta Soc. Sci. Fenn. Ser. B, Opera Biol. 7:20. 1942. Pistacia mutica var. cypricola (H. Lindb.) H. Lindb., Acta Soc. Sci. Fenn. Ser. B, Opera Biol. 7:22. 1946. TYPE: CYPRUS: presumably a Harald Lindberg specimen at $\mathrm{H}$.

Pistacia atlantica f. oxycarpa Zohary, Palestine J. Bot. Jerusalem Ser. 5:206. 1952. TYPE: AFGHANISTAN: Griffith 1269 (K).

Shrubs or trees, 3 - $15 \mathrm{~m}$ high; crown wide, rounded. Leaves deciduous, imparipinnate, 8 - $17.6 \mathrm{~cm}$ long, 5.2 $14 \mathrm{~cm}$ wide, membranaceous; petiole flattened; rachis narrowly winged. Leaflets (5-)7-11, 3 - $7 \mathrm{~cm}$ long, 5 - 20 $\mathrm{mm}$ wide, average ratio 3.5-1, opposite, lower subopposite, lanceolate, obtuse, minutely puberulent; rachis

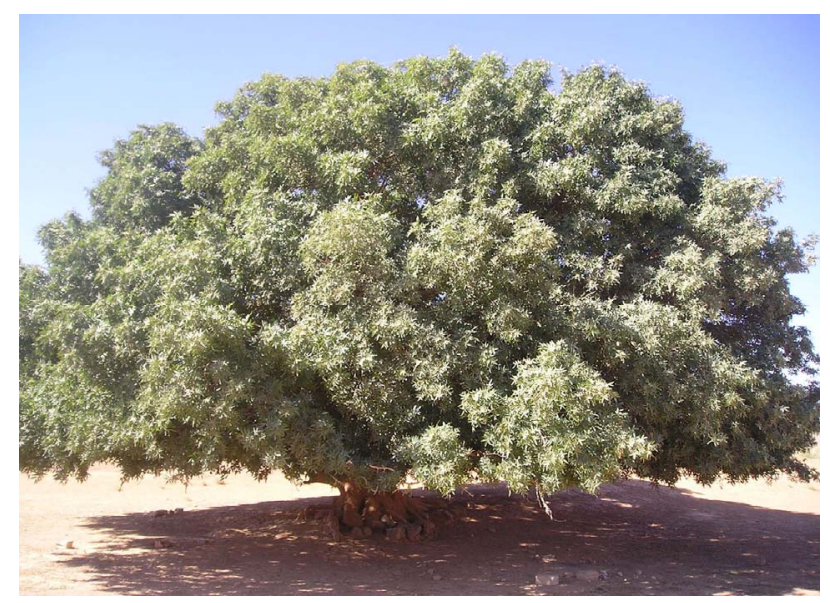

Figure 1. Pistacia. atlantica tree. The picture shows the species leaves. This species is located in Baqqawaeh, Safawi, Jordan. Bar Scale 1:1000. minutely puberulent; terminal leaflet 2.7 - $7 \mathrm{~cm}$ long, 5 $20 \mathrm{~mm}$ wide, same size or smaller than laterals. Staminate panicles to $7(-11) \mathrm{cm}$ long, often clustered, stout, branched from base, densely flowered, pubescent; flowers red. Pistillate panicles to $15 \mathrm{~cm}$ long, subterminal with leaves, stout, branched from base, minutely pubescent; flowers pinkish. Drupes many, red or reddish, 3 mm long, mesocarp fleshy, endocarp bony.

Phylogeny. $-P$. atlantica iswas close and sister to $P$. eurycarpa [7].

Phenology.-Flowering December, January, March to May; fruiting March to September.

Habitat and ecology.--Stony hillsides, steep dry slopes, limestone and sandstone hills; $30-2500 \mathrm{~m}$.

Use.-Iran: Galls common, "used for dyeing (black)" (Lace 3677, 3678).

Common names.-Betoum, Butum (Zohary 1952). Cyprus: Gum Tree, Trimithia (Ecoyomides 1019). Iraq (Kurdish): Kazwan (Gillett 7800, 7940). Libya: Batum (Keith 146), Bitam (Sandwith 2780; Sandwith \& Simpson 39730). Pakistan: Gwan (Ghafour \& Goodman 5164), Khanjak (27 Apr 1888, Lace).

Distribution.-From the Canary Islands, accross North Africa, to Greece, Turkey, and Cyprus, and through the Middle East and Saudi Arabia to Ukraine, Georgia, and Azerbaijan, and Afghanistan and Pakistan.

Specimens examined. AFGHANISTAN. Baluchistan: Khojak Pass, 04 Nov 1888, J.F. Duthie 8627 (BM). Heart: between Qala Charak and Chisht, $34^{\circ} 18^{\prime} \mathrm{N} 64^{\circ} 00^{\prime} \mathrm{E}, 1800$ m, 08 Apr 1962, K.H. Rechinger 19192 (MO). Konar: Asmar Barikot, 900 m, 1949, L. Edelberg 1639 (K). Paktia: Kurrum [Karram] Valley, Alisaí, 04 Apr 1879, J.E.T. Aitchison s.n. (BM, K). Shalina: May 19 1879, JETA 361 (BM, K). ALGERIA. Djelfa: $15 \mathrm{~km}$ N of Berriane, 800 m, 23 Jul 1982, Baxter et al. 4078 (E). Batna: NW foot of Col de Telmet, W of Batna, 1300 m, 21 May 1971, Davis 52568 (BM). Laghouat: Djebel Amour, between Aflou and El Ghicha, 1350 m, 04 Jun 1975, Davis 58745, (BM). Naama: Djebel Mekter, near Aïn Safra, 1600 - 2000 m, May 1913, E Hartert s.n. (BM). Mila: between Grarem and Mila, 200 m, 22 May 1975, Davis 58207A (BM). Saïda: Saïda, May 1858, G. Munby s.n. (K); near Saïda, 22 May 1852, B. Balansa 340 (K). Tlemcen: Tlemcen, 1867, G. Munby s.n. (K). Unknown region: 1803, Desfontaines s.n. (BM). ARMENIA. Szovits 46 (K); near Shvanidzov, Gerundara Valley, 03 May 1967, E. Gabrizhan \& P. Gambarian s.n. (K). AZERBAIJHAN. Zangelan: between Pircheran and Chari-Mushulag (cult.), 400 m, 09 May 1948, A. Grossheim et al. s.n. (MO); between Razadara and frontier with Armenia, 600 m, 10 May 1948, A. Grossheim et al. s.n. (MO). Unknown region: near Shamkhoz, $130 \mathrm{~m}, 22$ May 1982, A.K.Shvortsov s.n. (MO). CANARY ISLANDS. Gomera: Hermigua, 24 Apr 1845, E. Bourgeau 
812 (BM); La Roque, 600 m, Mar 1906, C.J. Pitard 545 (MO). Gran Canaria: E of Agaete, 100 m, 18 Apr 1981, Davis 67350 (E). Tenerife: Anaja Hills?, Cabrera s.n. (K); Sitio del Pardo, Orotara (cult?), 19 Jun 1899, R.P. Murray s.n. (K). CYPRUS. Kambyli: 520 ft, 27 Feb 1955, L.F.H. Merton 1988 (K). Neophytos: 1000 ft, 25 Jul 1967, S. Ecoyomides 1019 (K). EGYPT. Sharqiya: Tell-el-Kebîr, Jan 1913, R. Muschler s.n. (MO). GEORGIA. Dedoplistskaro: Vashlovani Forest Reserve, 41 ${ }^{\circ}$ 14'N 46²2'E, 13 May 1999, J.F. Gaskin 175, 192 (MO). GREECE. Crete: Gorges de Imbros, N of Chora Sfakion, 660 m, 09 May 1984, R. Deschamps 9093 (MO). Dodekanisos: Rhodes, near the Acropolis, 18 Apr 971, C.C. Townsend 71/37 (K). Drama: $4 \mathrm{~km}$ from Paranestion toward Drama, $41^{\circ} 15^{\prime} \mathrm{N} 24^{\circ} 28^{\prime} \mathrm{E}, 220 \mathrm{~m}, 22$ Jun 1988 , Strid et al. 27266 (E). Voreio Aigaio: Lesbos, Apr 1968, O.M. Bedford s.n. (BM). IRAN. Baluchistan va Sistan: Dilkuna, 6000 ft, 30 Mar 1888, J.H. Lace 36777, 3678 (E); 74 - $100 \mathrm{~km}$ from Khash-Iranshar road toward Bazman, 1600 m, 29 Apr 1977, K.H. Rechinger 54955 (E); between Khash and Iranshahr, $1500-1600$ m, 16 - 17 May 1948, K.H. Rechinger \& F. Rechinger 4033 (MO); $17 \mathrm{mi} \mathrm{W}$ of Saravan toward Suran, $3900 \mathrm{ft}, 18 \mathrm{Mar}$ 1971, C. Grey-Wilson \& T.F. Hewer 202 (K). Fars: 25 km N of Firusabad, 1400 m, 08 Nov 1963, M.L. Grant 15032 (MO); 20 - 30 km W of Qir, 1300 m, 02 Apr 1974, Davis \& Bokhari 56337 (K, MO). Ilam: near Nargesi, 12 May 1975, T. Antonio et al. 256 (MO). Kerman: between Dasht-e-Lut and Jaz Murian, $29^{\circ} 04^{\prime} \mathrm{N} 57^{\circ} 57^{\prime} \mathrm{E}, 1850 \mathrm{~m}$, 19 Apr 1972, J. Léonard 5630 (K); near Khabr, W side of Kuh-e-Khabr, $28^{\circ} 51^{\prime} \mathrm{N} 56^{\circ} 23^{\prime} \mathrm{E}, 2300 \mathrm{~m}, 02$ May 1975 , B.S. Parris 75.298 (E). Khorasan: near Jangal, $1500 \mathrm{~m}$, 01 Jun 1963, G. Popov 233 (BM). IRAQ. Arbil: Sefin Dagh, 11 Jul 1972, F. Karim 39400 (K). As Sulaymaniyah: Pir Omar Gudrum, above Qarachitan, 19 Apr 1947, J.B. Gillett 7800 (K); Qaradagh, 1000 m, 01 Jul 1948, G. Chapman 12312 (K); Qara Dagh Mt., 900 - 1750 m, 23 Apr 1947, J.B. Gillett 7940 (K). Ninawa: Iskeftendiwar, near Shaikh Adi, 2500 ft, 14 Jul 1933, Crambquest 3666 (K). JORDAN. Ajloun: Ajloun, 877 m, 24 May 2004, M. G. AL-Saghir JOR3 (VPI); $960 \mathrm{~m}, 24$ May 2004, M.G. AL-Saghir JOR4 (VPI); 1092 m, 24 May 2004, M.G. ALSaghir JOR6 (VPI); Bair Al Dabaghat, Shobak, 04 Jun 2004, M.G. AL-Saghir JOR 22, JOR 23 (VPI); $771 \mathrm{~m}, 04$ Jun 2004, M.G. AL-Saghir JOR 20, JOR 21 (VPI); Baqqawaeh, Safawi, 02 Jun 2004, M.G. AL-Saghir JOR15a (VPI); Jebel Salab, W of Ain Labaha, $30^{\circ} 42^{\prime} \mathrm{N} 35^{\circ} 36^{\prime} \mathrm{E}$, 1530 m, 24 Apr 1963, J.B. Gillett 15844 (K); Wadi above Khasr Amra, 16 Sep 1965, J. Hemsley s.n. (K); Nayma, 1017 m, 29 May 2004, M.G. AL-Saghir JOR8, JOR10 (VPI); near Qasr 'Amra, Azraq Oasis, 25 Apr 1974, L. Boulos et al. 7084 (BM, K); Qasr 'Amra, $25 \mathrm{~km} \mathrm{SW}$ of Azraq Oasis, 14 May 1976, V. Täckholm et al. 9168 (K); Umm Qays, 275 m, 29 May 2004, M.G. AL-Saghir JOR1
(VPI); Wadi AL-Botom, Azraq, 02 Jun 2004, M.G. ALSaghir JOR16 (VPI); Wadi AL-Botom, Sahaab, 02 Jun 2004, M.G. AL-Saghir JOR17 (VPI); 610 m, 02 Jun 2004, M.G. AL-Saghir JOR18 (VPI); 617 m, 02 Jun 2004, M.G. AL-Saghir JOR19 (VPI). LIBYA. Beni Snassen: $10 \mathrm{~km}$ SSW of Berkane, $34^{\circ} 50^{\prime} \mathrm{N} 2^{\circ} 21^{\prime} \mathrm{W}, 630 \mathrm{~m}, 29$ Oct 1993 , S.L. Jury \& T.M Upson 13056 (BM); Gebel Nefoussa, Ain Zarga, 600 - 700 m, 17 Mar 1970, Davis 49685 (E); near Iefren, 700 m, 15 Mar 1970, Davis 49540 (E, K); Oroban, N of Senam Ulaad Brekk, 900 m, 11 May 1957, H.G. Keith 146 (K). Gharyan: Gharyan plateau, near El Rumia, 700 - 750 m, 25 Apr 1939, N.Y. Sandwith 2780 (K). Yafran: Jefren [Yafran], near Rumîa, 25 Apr 1939, N.Y. Sandwith \& N.D. Simpson 39730 (BM). MOROCCO. Wiwam to Jouner Oum En Rbie, 1700 m, 18 Jun 1991, J. Lewalle 13550 (MO). Agadir: Immouzer Valley, N of Agadir, 28 Mar 1972, D. Bramwell et al. 280 (K, MO). Er-Rachidia: near Gorge du Ziz, N of Er-Rachidia toward Midelt, $32^{\circ} 06^{\prime} \mathrm{N} 04^{\circ} 23^{\prime} \mathrm{W}, 1110 \mathrm{~m}, 12 \mathrm{Jul}$ 1997, S. L. Jury et al. 17804 (BM). Marrakech: $63 \mathrm{~km}$ from Asni toward Tizi-n-Test, $30^{\circ} 54^{\prime} \mathrm{N} 08^{\circ} 20^{\prime} \mathrm{W}, 1650 \mathrm{~m}, 20 \mathrm{Jul}$ 1989, M. Ait Lafkih et al. 780 (BM); $1 \mathrm{~km} \mathrm{NNE} \mathrm{of} \mathrm{Ijou-}$ kak, $45 \mathrm{~km} \mathrm{SW}$ of Asni, $31^{\circ} 01^{\prime} \mathrm{N} 08^{\circ} 09^{\prime} \mathrm{W}, 1170 \mathrm{~m}, 10$ Jul 1993, S.L. Jury \& L.S. Springate 11959 (BM, K); 65 $\mathrm{km}$ from Marrakech toward Tizi-n-Tichka, $31^{\circ} 29^{\prime} \mathrm{N} 07^{\circ}$ 26'W, 1510 m, 10 Jul 1987, S.L. Jury et al. 9157 (BM); Monildikht, between Tizi n'Test and Ichoukak, $5000 \mathrm{ft}$, 26 Jun 1936, E.K. Balls 2938 (BM). Rabat-Sale: 12 km SE of Aïn-el-Aouda, 09 May 1968, G. Mines \& J. Veilex s.n. (MO). Taroudanut: Oued Issen, 16 Jun, H. Lynes $13 a$ \& $13 b$ (BM). Tiznit: $11 \mathrm{~km}$ from Tafraout toward Titeki, 1250 m, 28 Mar 1969, P. \& J. Davis 48863 (E); above Tafraout toward pass to Ait-Baha, $1200 \mathrm{~m}, 30 \mathrm{Mar}$ 1972, Davis 53888 (BM); Tazerwalt, Ihlwane, 800 m, 27 Mar 1981, J. Lewalle 9731 (BM, MO). PAKISTAN. Balochistan: Fort Sandeman [Zhoh], $4500 \mathrm{ft}, 17 \mathrm{Jun}$ 1976, I. Dar \& A. Mohd 5 (MO); Gwál, 6000 ft, 27 Apr 1888, J.H. Lace s.n. (E); Hazarganji, near Quetta, 23 Aug 1962, R.R. Stewart 831 (K); Juryi Pass, 2849'N 6639'E, 11 May 1990, A. Ghafoor \& S.M. Goodman 5164 (F); between Loralai and Qila Saifullah, 19 May 1984, S. Omar \& A. Ghafoor 1635 (MO). North West Frontier Province: Ayun, 26 Jul 1976, M. Shah et al. 2227 (MO); Birmoghlasht, 7 - 8000 ft, 02 Aug 1954, M.A. Saddiqui \& A. Rahman 26767 (BM); Maroi, between Chitral and Mastuj, 6000 ft, 28 May 1958, J.D.A. Stainton 2532 (BM). PALESTINE. Asher, $29 \mathrm{Feb}$ and 15 May 1913, F. Myers 9239 (K); Jerusalem, 29 Mar and 15 May 1913, J. E. Dinsmore 9239 (F); 20 Apr 1903, F. Meyers 712 (F); Lower Galilee, between Yagur and Tiv'on, 01 Jun 1951, M. Zohary 441 (BM, K, MO). SAUDI ARABIA. Hejaz: near Suq ar Rubu, S of Taif, 7000 ft, 07 Apr 1977, I.S. Collenette 258 (K). Mintaqat Jizan. Al-Khadra to near Ghamid-Al-Zinad, 15 Jul 1976, J. Dwyer \& A. El-Sheikh 
13772 (MO). Tabuk: Jabal Dabbagh, $100 \mathrm{~km} \mathrm{SW}$ of Tabuk, 3000 ft, 03 Mar 1984, I.S. Collenette 4785 (K). SOUTH AFRICA. Ganteng: Pretoria (cult.), 21 Sep 1961, coll. unknown (K). TUNESIA. Sidi Bouzid: Marabat Sidi Khaled, between Sidi Bouzid and Maknassi, 01 Apr 1968, L. Boulos 273 (MO).TURKEY. Antalya: Seldsjuk bridge about $5 \mathrm{~km}$ E of Serik $30 \mathrm{~m}, 23 \mathrm{Apr}$ 1959, E. Hennipman et al. 638 (K). Icel: Yenege [Yenice], between Tarsus and Adana, 50 ft, 10 Apr 1934, E.K. Balls 712 (BM). Istanbul Ili: Western Thracia, Tarabya, 50 m, 23 Apr 1961, K.H. Rechinger 21958 (MO). Karabak: Igdir to Safranbolu, 350 m, 02 Aug 1962, Davis et al. 38799 (K, MO). Mugla Ili: Bodrum, Musgebi [Ortakent] to Karatoprak, 50 - 100 m, 12 Apr 1965, Davis 41003 (K). UKRAINE. Republika Krym: Tauria, near Nikita, 100 m, 25 Jul 1967, A.K. Shvortsov 82 (MO). ZIMBABWE. Harare: Harare, Greenwood Park (cult.), 22 Oct 1937, R.J. Finlay m140/37 (BM).

\subsubsection{Pistacia chinensis Bunge, Enum, Pl. China Bor. 15. 1833.}

Type: "Peking leg. Bunge, in Herb, Petropol." [LE] (Zohary 1952 , p. 215).

\subsubsection{Pistacia chinensis subsp. chinensis}

Pistacia formosana Matsum., Bot. Mag. (Tokyo) 15:40. 1901. TYPE: not designated.

Pistacia chinensis f. latifoliata Loes. in Gilg. \& Loes., Beih. Bot. Jahrb. Syst. 34, 75:49.1904. TYPE: not designated.

Pistacia philippinensis Merr. \& Rolfe, Philip. J. Sci. 3: 107.1908. TYPE: not designated. Zohary (1952, p. 215) refers to Vidal 1825 from Benguet Province, Luzon, Philippines (K) as an "isotype".

Trees, 4 - $20 \mathrm{~m}$ high. Leaves deciduous, paripinnate, 8.2 - $23 \mathrm{~cm}$ long, 8 - $20 \mathrm{~cm}$ wide, membranaceous; petiole angled or flattened, minutely reddish-pubescent; rachis not winged, minutely reddish-pubescent. Leaflets 8 14, 4 - $10 \mathrm{~cm}$ long, 9 - $24 \mathrm{~mm}$ wide, average ratio 4.1-1, lanceolate, attenuate, apiculate, uppermost opposite, lower usually subopposite, midvein and margin pubescent, becoming glabrate. Staminate panicles to $4 \mathrm{~cm}$ long, manyflowered, catkin-like when young, pubescence reddishbrown. Pistillate panicles to $17 \mathrm{~cm}$ long, many-flowered, stout, branched from base, minutely puberulent with reddish-brown trichomes, which are longer on younger inflorescences. Drupes green turning red, drying grayish, apiculate, $5 \mathrm{~mm}$ long.

Phylogeny. $-P$. chinensis is sister to $P$. falcata and $P$. integerrima [7].

Phenology.-Flowering April, September, and October; fruiting March, and May to November.

Habitat and ecology.-On limestone; 200 - $2500 \mathrm{~m}$.

Uses.-China: "large tree, young leaves of which are eaten by the Chinese" (A. Henry 340); "fruit edible (for oil)" (Sino-British Expedition to Cangshan 125).

Common names.-China: Huang-lien-shur or Huangm-ya shu [10]; Chinese pistachio [22]; Huang-lien-ya (Henry 3402). Taiwan (Japanese): Ransinboku (Matuda, 07 Jan 1918).

Distribution.- Native to China, Taiwan, and the Philippines.

Specimens examined. CHINA. Beijing: near Peking [Beijing], 1867, S.W. Bushell s.n. (F); S.W. Williams s.n. (BM); Tehé-hai, 2500 m, Nov, Léveillé s.n. (E); Tongtehouen [Tongtian? $10 \mathrm{~km} \mathrm{~N}$ of Ganzhou?], $2500 \mathrm{~m}$, Apr, Léveillé s.n. (E). Fujian: H.H. Chung 6228 (F); Tiger Hill, Amoy Island, 30 Apr 1923, H.H. Chung 1445 (K). Gansu: Shuijiawan, Wen Xian, 630 m, 26 May 1992, X. Wang 159 (MO). Guangdong: Aug 1887, C. Ford 308 (K); Lian Xian, 02 Oct 1950, T. Peixiang 59618 (MO); S of Linchow, Yang Shan District, Jul-Sep 1932, T.M. Tsui 495 (F); near Lo Tsi Tsui, Lin District, 14 Oct 1918, N.C. Lam s.n. (MO); Ruyuan, 23 Oct 1956, C. Wang 42275 (MO); Yunfu Xian, 22 May 1934, C. Wang 37030 (MO). Guangxi: Fushi Town, Liuliao, $20 \mathrm{~km} \mathrm{~S}$ of Ron- gan, 500 m, 10 May 1997, P.J. Cribb et al. 485 (K); Kweilin, 220 m, 1979, P.P. Wan \& K.S. Chow 79169 (BM, K, MO); Lingyun Xian, 28 Jul 1937, L. Xinqi 28776 (MO); Loh Hoh Tsuen, Ling Yün Hsien, 1150 m, 18 May 1933, A.N. Steward \& H.C. Cheo 443 (BM). Guizhou: Holupei, Tungtze, 400 m, 17 May 1930, Y. Tsiang 4898 (BM). Hong Kong: Kowloon, Tai-Hung Tung, 27 Aug 2002, S.Y. Hu \& K.H. Yung 514 (K, MO). Hunan: Sing-Ch'u, Sintien Hsien, 600 m, 23 Aug 1935, C.S. Fan \& Y.Y. Li 374 (BM). Hubei: W Hupeh [Hubei], Apr 1907, E.H. Wilson 380 (BM, E); Oct 1907, coll. un- known (BM). Ichang [Yichang], Oct 1887, A. Henry 3402 (K). Jiangsu: 28 Aug 1995, W. Wenxiang 9963 (MO); I-hing, 10 Oct 1979, W.Z. Fang 7939 (MO); Jiangsu Botanical Institute (cult.), 08 Nov 1982, K. Yao 8535 (MO); Yun-tai-shan, Lian-yun-gang (cult.), 200 m, 22 Sep 19, K. Yao 848481 (MO). Shandong: Chefoo [Chi-Fu; Yantai], 17 Sep 1880, F.B. Forbes s.n. (BM, 2 colls.); Ching Shan, Lao Shan, 600 m, 20 Jul 1930, C.Y. Chiao 2834 (E); Pao Shan, near Tsinanfu, 200 m, 20 Sep 1930, C.Y. Chiao 3152 (F); Tsing-tao, 29 May 1979, C.R. Lancaster s.n. (BM). Shanghai: near Shanghai, Apr and May 1887, W.R. Carles 275 (E, K); 1873, C. Cooper 275 (K). Sichuan: Bai Di Cheng, 280 m (cult.), 06 Jun 1996, C. Zhiduan et al. 96099 (MO); Kuan-hsien, 08 Apr 1938, W.P. Fang 12132 (BM); near Techang, 03 Apr 1914, C. Schneider 710 (K); Tianci Forest Farm, 960 m, 21 May 1996, C. Zhiduan et al. 960417 (MO). Yunnan: Cao Xi Temple, Anning, 42 km W of Kunming, 2025 m, 29 Apr 1981, Sino-British Expedition to Cangshan 126 (E, K), 127 (E, $\mathrm{K})$; near Guylieangbien and Loulantchean, 30 May 1897, E. Bodinier 2304 (E); Liao-mi-lan, near Cali, 19 Jul 1895, 
Delavay 1953 (K); Lichiang Range, 1933, H. D. McLaren's collectors 241c(BM); Lunan Xian, Chang- hu, $24^{\circ} 43^{\prime} \mathrm{N} 103^{\circ} 25^{\prime} \mathrm{E}, 1850$ - $2000 \mathrm{~m}, 02$ Aug 1984, Sino-American Botanical Expedition 1581 (MO); Merchse, $4500 \mathrm{ft}$, A. Henry 10254A (E); S. loc., 06 Mar 1993, T. Ceming 93159 (MO); S. loc., 700 m, 23 Sep 1958, L. Hanlin 9938 (MO); S. loc., 21 Aug 1957, Y.Y. Ho 27651 (MO); 09 May 1982, Y. Huagu 730 (MO); S. loc., 16 Jun 1936, Z. Jixin 205 (MO); 22 Jul 1978, Jurong Group of Nanjing Foresty University Expedition 7064 (MO); 19 Nov 1931, C. Naingu 42986 (MO); 19 Oct 1979, L. Qinghua 5072 (MO); S. loc., Z. Shaoyao 2218, NA (MO); S. loc., 05 Jul 1958, Z. Shaoyao 2381 (MO); S. loc., 680 m, 27 May 1962, L. Tianwei 230 (MO); S. loc., 16 Jun 1993, L. Tianwei \& Z. Zhaofen 258 (MO); S. loc., 29 Aug 1958, H. Xianyu 30203 (MO); S. loc., 07 Feb 1996, H. L. Yin 6062 (MO); S. loc., 08 May 1996, L. Yongshan 96296 (MO); S. loc., 28 Aug 1996, L. Zhao 53 (MO); S. loc., 12 Jun 1984, L. Zhiyou 72 (MO); S. loc., 300 m, 24 Oct 1963, L. Linhan \& H. Guanzhou 16476 (MO); S. loc., 15 Aug 1959, Z. Zhiying 14018 (MO). Zhejiang: Ningpo [Ningbo] District, Aug 1884, Cooper s.n. (K); Ou-Kiang, S of Ping Yang, 21 Jun 1924, R.C. Ching 1920 (K). Unknown region: Kew Kiang [173 $\mathrm{mi} \mathrm{N}$ of Han-Kow], 1873, D. Shearer s.n. (K); Western China, dry valleys, Sep 1903, E.H. Wilson 3363(K). PHILIPPINES. Benguet: Baguio, Mar 1907, A.D. E. Elmer 8779 (BM, MO). Ifugao: Bontoc, May 1923, Zschokke \& Laraya 29400 (BM). Ilocos Sur: Oct 1915, P. de la Peña 24687 (F). TAIWAN. Hualien: Hsiulin, Holiu, $24^{\circ} 10^{\prime} \mathrm{N} 121^{\circ} 30^{\prime} \mathrm{E}$, 450 m, 23 Apr 1994, B.J. Conn \& C.-I Peng 4089 (MO). Nanshan: Ilan Hsian, Chi-Lan Shan, 1200 m, $24^{\circ} 38^{\prime} \mathrm{N}$ $121^{\circ} 28^{\prime} \mathrm{E}, 11$ Nov 1993, S. Clarke et al. 339 (E). Nantou: Hsinyi Hsiang, Chunta Forest Road, $23^{\circ} 39^{\prime} \mathrm{N} 120^{\circ} 52^{\prime} \mathrm{E}$, 625 - 695 m, 28 Sep 1994, K.Y. Yang et al. 226 (MO). Tainan: Nanhsi Hsiang, $150 \mathrm{~m}, 23^{\circ} 14^{\prime} \mathrm{N} 120^{\circ} 31^{\prime} \mathrm{E}, 04$ Apr 1993, W.P. Leu \& W.-M, Wang 2011 (E); Shishto, E of Kousha, 11 Jul 1912, W.R. Price 858bis (K). Taipei: Taipei City, Taipei Botanical Garden (cult.?), $25^{\circ} 03^{\prime} \mathrm{N}$ $121^{\circ} 31^{\prime} \mathrm{E}, 17$ May 1996, C. Liou et al. 148 (E); Takubun, Jan 1918, E. Matuda s.n. (BM). USA. California: Berkeley (cult.), 09 Sep 1942, N.F. Bracelin 2283 (F); 25 Jun 1944, coll. unknown 2597? (F). Texas: Houston (cult.), "Ju" 1938, coll. unknown (F).

\subsubsection{Pistacia chinensis Bunge subsp. falcata (Becc. ex Martelli) Rech. f., Fl. Iranica 63:8.1969}

Basionym: Pistacia falcata Becc. ex Martelli, Fl. Bogos. 24. 1886. Rhus falcata (Becc. \& Martelli) Penz., Atti Congr. Bot. Genova 337. 1893. Pistacia chinensis var. falcata (Becc. ex Martelli) Zohary, Palestine J. Bot. Jerusalem Ser. 5:217. 1952. Type: ERITREA: Keren, sul Monte, Deban frai 1400 - 1700 m, 1870, Beccari 273 (HOLOTYPE: FI).
Trees, 4 - $10 \mathrm{~m}$ high; twigs minutely brownish-yellow pubescent. Leaves deciduous, imparipinnate, $13-21 \mathrm{~cm}$ long, $11.4-18.2 \mathrm{~cm}$ wide, membranaceous; petiole flattened; rachis not winged. Leaflets $11-15,5.2-9.1 \mathrm{~cm}$ long, $1-2 \mathrm{~cm}$ wide, average ratio 5.5-1, narrowly lanceolate, falcate, attenuate, glabrous, opposite to subopposite below; terminal leaflet commonly present, 2 - 4 $\mathrm{cm}$ long, $5-11 \mathrm{~mm}$ wide, smaller than laterals or reduced. Staminate panicles to $8 \mathrm{~cm}$ long, branched from base, branches spike-like, trichomes pale; flowers yellow, turning red. Pistillate panicles to $15 \mathrm{~cm}$ long, not branched from base, stout. Drupes to $7 \mathrm{~mm}$ long, greenish-white, turning reddish.

Phylogeny. $-P$. falcata is sister to $P$. chinensis and $P$. integerrima [7].

Phenology.-Flowering February and April; fruiting February, April to August, and November.

Habitat and Ecology.-Savannahs, on calcareous or volcanic soils and old or recent lava flows; $1100-2600$ $\mathrm{m}$.

Common Names.-Somalia: Sisaye (Glover \& Gilliland 1114), Sisei (Glover \& Gilliland 1164).

Distribution.-Egypt, Saudi Arabia, and Yemen, to Eritrea, Ethiopia, and Somalia.

Specimens examined. EGYPT. Al Bahr Al Ahmar: Wadi Haikwal, Gebel Elba, 17 Sep 1936, M. Drar 57/ 884 (K); South Galala, Wadi Rigba, 520 m, 04 Apr 1944, P.H. Davis s.n. (BM). ERITREA. Hamasien: Monte Zagher, 2600 m, 20 May 1902, A. Pappi 5308 (BM, F). ETHIOPIA. Afar: about $50 \mathrm{~km} \mathrm{SW}$ of Awash Station, 700 m, 05 Apr 1966, W.J.J.O. de Wilde 10484 (MO). Amhara: Fantalle Volcano crater, Awash National Park, $08^{\circ} 59^{\prime} \mathrm{N} 39^{\circ} 55^{\prime} \mathrm{E}, 1500 \mathrm{~m}, 16 \mathrm{Feb} 1970$, J.J.F.E. de Wilde 6350 (MO); Garibaldi Pass, $28 \mathrm{~km} \mathrm{~W}$ of Metahana, 1100 m, 10 Feb 1974, J.W. Ash 2360 (MO); just W of Garibaldi Pass, $08^{\circ} 49^{\prime} \mathrm{N} 39^{\circ} 43^{\prime} \mathrm{E}, 1260 \mathrm{~m}, 01 \mathrm{Feb} 1963, W$. Burger 3616, 3618 (F); $29 \mathrm{~km}$ on Metahana to Nazret road, $8^{\circ} 52^{\prime} \mathrm{N} 39^{\circ} 45^{\prime} \mathrm{E}, 1450 \mathrm{~m}, 21$ May 1980 , M. Thulin et al. 3875 (MO). Oromia: Darro Mts, 18 Nov 1894, A. Donaldson Smith 266 (BM); $5 \mathrm{~km}$ E of Wolenchetti [Welench iti], 804'N 39 30'E, $1300 \mathrm{~m}, 16$ Feb 1965, W. Burger 3695 (F). SAUDI ARABIA. Al-Bahah: Beni Ghamid, 6500 ft, 14 Jun 1946, W. Thesiger s.n. (BM). Asir: $5 \mathrm{~km} \mathrm{~S}$ of Abha, $18^{\circ} 11^{\prime} \mathrm{N} 42^{\circ} 29^{\prime} \mathrm{E}, 2050 \mathrm{~m}, 04$ Aug 1982, U. Baierle \& P. König s.n. (E); Taif, 2000 m, 1985, A.R. Pittaway s.n. (BM). SOMALIA. Sanaaq: Tabah Gap [Pass], 05 Jul 1945 P.E. Glover \& H. B. Gilliland 1114 (BM). Woqooyi Galbeed: Gan Libah, $5300 \mathrm{ft}, 25$ Jul 1945, P.E. Glover \& H.B. Gilliland 1164 (BM). YEMEN. San'a: Jebel Bura, near Maghraba, 1700 m, 27 May 1977, J.R.I. Wood 1642 (BM); Djebel Dhûrân, 1931, H. van Wissmann 1849 (BM); Jebel Melhan, between Markah and Maghraba, 1800 m, 02 Jun 1978, J.R.I. Wood 2372 (BM). 


\subsubsection{Pistacia chinensis Bunge subsp. integerrima}

(J.L. Stew. ex Brandis) Rech. f., Fl. Iranica 63:8. 1969

Basionym: Pistacia integerrima J.L. Stew. ex Brandis, Forest Fl. NW. India 122. t. 22. 1874. Pistacia chinensis var. integerrima (J.L. Stew. ex Brandis) Zohary, Palestine J. Bot. Jerusalem Ser. 5:216. 1952. Type: not designnated, nor are any specimens cited (lectotype, here designated: t. 22, Brandis 1874).

Rhus kakrasingee Royle, Ill. Bot. Himal. Mts. 5:175. 1835. nom. nud.

Rhus integerrima Wall., Numer. List 8474.1847. nom. nud.

Pistacia khinjuk var. stocksii Engl. in A. \& C. DC., Monogr. Phan. 4:291. 1883. TYPE: not designated.

Lentiscus kakrasingee (Royle) Kuntze, Revis. Gen. Pl. 1:152.1891.

Trees, 6 - $8 \mathrm{~m}$ high. Leaves deciduous, usually paripinnate, $11-22 \mathrm{~cm}$ long, $12-23 \mathrm{~cm}$ wide, membranaceous; petiole angled or flattened; rachis not winged, reddish-pubescent when young, becoming glabrate. Leaflets 6-(9)-10, 6 - $11.5 \mathrm{~cm}$ long, $2-4 \mathrm{~cm}$ wide, average ratio 3.4-1, opposite to subopposite, broadly lanceolate, attenuate, glabrous. Staminate panicles to $10 \mathrm{~cm}$ long, branched from base, branches spike-like, reddish-pubescent, young parts especially pubescent. Pistillate pancles to $16 \mathrm{~cm}$ long, branched from base, stout in fruit. Drupes red.

Phylogeny. $-P$. integerrima was sister to $P$. chinensis and $P$. falcate [7].

Phenology.-Flowering March and April; fruiting April to June.

Habitat and ecology.-800 - $2900 \mathrm{~m}$.

Common names.-Gusgu, Kakrangche, Kakrat, Kakring, Kakroi, Kanroi, Masua (Afghanistan), Sarawan, Shue, Tungu [10]. Pakistan: Kangar (Barrett 64).

Distribution.-Armenia, Afghanistan, Pakistan, and Nepal.

Specimens examined. AFGHANISTAN. Paktia: Kurrum [Kurram] Valley, near Shálizán, 06 Apr 1879, J.E.T. Aitchison 38 (K). ARMENIA. Szovits 2510 (BM). NEPAL. Purini, Pultanto Dara (cult.?), $7000 \mathrm{ft}, 21$ Apr 1952, O. Polunin et al. 802 (BM).PAKISTAN. Kashmir: Bhimber, 2500 ft, 28 Jun 1876, C.B. Clarke 28117 (K); M'Tilla, Cabilica (cult.), 27 Mar 1893, Aitchison 4 (K). North West Fronter Province: Bromboret Gol, Chitral, $35^{\circ} 41^{\prime} \mathrm{N} 71^{\circ} 38^{\prime} \mathrm{E}, 6800 \mathrm{ft}, 15$ May 1958, S.A. Bowes Lyon 651 (BM); Hazara, Bisian, 3000 ft, 24 Apr 1978, M. Shah \& D. Khan 1808 (K); Hazara, Manchora, 14 May 1976, M. Shaukat \& N. Ahmad 266 (K); Jaunsar District, Thadyar, 8000 ft, May 1891, J.S. Gamble 23024 (K); Jaunsar, Kachanu, 4000 ft, Mar and Jun 1890, K. Nand 214 (BM); Swat, Miadam, 6000 ft., 29 May 1976, M. Shah \& D. Khan 1073 (MO). Punjab: Barrett 21602 (K);
Banahul, 05 Nov 1848, J. Thomson s.n. (K); Harara District, 3000 - $5000 \mathrm{ft}, 2003$ ?, Barrett 84 (K); Rawalpindi, Margalla Hills, 14 Apr 1976, I. Dar et al. 1 (MO). Unknown region: $W$. Roxburgh s.n. (BM); Ramaon, Jun 1845, J. Thomson 1213 (K); Karrot [Kanof], 2300 ft, 07 May 1969, S. ul-Abedin 2775 (MO, 2 colls.).

\subsubsection{Pistacia eurycarpa Yalt., Notes Roy. Bot. Gard. Edinb. 28:11. 1967}

Type: TURKEY: Prov. Bitlis, Bilitus gorge below Tutu, S of Kambos (Humus) Da., 1250 m, 16 Aug 1956, McNeill 600 (holotype: E).

Pistacia atlantica var. kurdica Zohary, Palestine J. Bot. Jerusalem Ser. 5:206. 1952. Pistacia atlantica subsp. kurdica (Zohary) Rech. F., Fl. Iranica 63:5. 1969. TYPE: not designated.

Shrubs or small trees, to $5 \mathrm{~m}$ high. Leaves deciduous, usually imparipinnate, $10.2-18.2 \mathrm{~cm}$ long, $9-13.5 \mathrm{~cm}$ wide, membranaceous; petiole flattened; rachis narrowly winged. Leaflets (1-3-) 5 - 7, $4.5-6.5 \mathrm{~cm}$ long, 1.5 - 3.8 $\mathrm{cm}$ wide, average ratio 2.3-1, lanceolate, obtuse, puberulent, margin ciliated to glabrous; terminal leaflet $5.2-8$ $\mathrm{cm}$ long, $2.5-4 \mathrm{~cm}$ wide. Staminate panicles not seen. Pistillate panicles to $18 \mathrm{~cm}$ long, branched from above base, stout. Drupes bright red, wider than long, to $7 \mathrm{~mm}$ long and $8 \mathrm{~mm}$ wide.

Phylogeny.-P. eurycarpa is close and sister to P. atlantica [7].

Phenology.-Flowering season unknown; fruiting May to August, October.

Habitat and ecology.-Stony slopes, shale or limestone; $1400-1800 \mathrm{~m}$.

Uses.-Turkey: "Fruits eaten" (Sperling et al. 6787).

Common names.-Badwar, Butum, Gazwan, Giwan, Habbat, Khadra, Khayak (Zohary 1952). Turkey: Derekhti Sakhus (Loftus, 01 Aug 1851); Fistik (Sperling et al. 6787); Wann (Kurdish) (Loftus, 01 Aug 1851).

Distribution.-Turkey, Armenia, Iraq, Iran, Afghanistan, and Pakistan.

Specimens examined. AFGHANISTAN. 1884-1885, $J$. E.T. Aitchison 1039 (BM). Farah: $60 \mathrm{Km}$ SSW of Farahbad, 1600 m, 15 Jul 1969, J.S. Andersen \& I.C. Petersen 434 (K). ARMENIA. Artashatskii region, between Arazdayn and Kiarkii, 26 Jun 1969, A.L. Takhtajan s.n. (BM). IRAN. Fars: S shore of Daryacheh-e Bakhtegan,

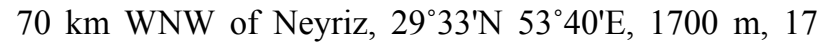
Jun 1977, M.H. Bokhari \& J.R. Edmondson 2093 (E); 3 km W of Saadatabad, 1800 m, 22 May 1964, M.L. Grant 15835 (MO). Gharbi: $25 \mathrm{~km}$ from Sardasht toward Baneh, 1400 m, 21 Oct 977, Fliegner \& Simmons 486 (K). Kermanshah: Tauk i Girrah, near Kirrind, 01 Aug 1851, W. K. Loftus s.n. (BM, 2 colls.). Kordestan: $101 \mathrm{~km} \mathrm{~S}$ of Baneh toward Marvan, 1700 m, 22 Oct 1977, Fliegner \& Simmons 501(K); between Sardasht and Baneh, $1500 \mathrm{~m}$, 
09 Jul 1974, K.H. Rechinger 491441974 (K). IRAQ. Arbil: Jebel Baradost, near Diana Rawandiz, 28-29 Jun 1934, H. Field \& Y. Lazar 942, 944 (F). Arbil \& Dahuk [Erbil \& Duhok]: Serv-i-Rust, 5300 ft, 07 Aug 1956, B. R. Haley102 (BM). As Sulaymanigah: Penjuin, 29 May 1932, B.P. Urazov s.n. (BM); Penwyn [Penjuin], 1600 m, 28 Aug 1953, E. Guest 12944 (K). Ninawa: Jebel Khatchra, near Balad Sinjar, 05 Jun 1934, H. Field \& Y. Lazar 652, 654 (F); Sheikh Adi, near Ain Sifni, 13 Jun 1934, $H$. Field \& Y. Lazar 714 (F). PAKISTAN. North West Frontier Province: Markanda, $6000 \mathrm{ft}, 20$ May 1895, S.A. Harris 16013 (BM). TURKEY. Hakkari: $9 \mathrm{mi} \mathrm{S}$ of Hakkari/Yuksekova road junction, 1550 m, 08 Jun 1966, Davis 44665 (E); above Zap River, $39 \mathrm{~km} \mathrm{~N}$ of Hakkari, $37^{\circ} 53^{\prime} \mathrm{N} 44^{\circ} 02^{\prime} \mathrm{E}, 1530 \mathrm{~m}, 19$ Jul 1985, C.R. Sperling, et al. $6787(\mathrm{E})$.

\subsubsection{Pistacia khinjuk Stocks, Hooker's J. Bot. Kew Gard. Misc. 4:143. 1852}

Type: PAKISTAN: Balochistan ("Beloochistan”), 1849, Stocks 719 (K).

Pistacia acuminta Boiss. \& Buhse, Nouv. Mém, Soc. Imp. Naturalistes Moscou 12:53. 1860. TYPE: not designated.

Pistacia khinjuk var. populifolia Boiss., Fl. Orient. 2:7. 1872. TYPE: AFGHANISTAN: Heart, between Heart and Tabas (Iran), 1858, Bunge (HOLOTYPE: G).

Pistacia khinjuk var. heterophylla Engl. in A. \& C. DC. Monogr. Phan. 4:290. 1883. TYPE: not designated.

Pistacia khinjuk var. glabra Schweinf. ex Engl. in A. \& C. DC. Monogr. Phan. 4:292. 1883. TYPE: EGYPT: middle desert, eastern part, W. Rigbe (HOLOTYPE: B).

Pistacia khinjuk var. glaberrima Schweinf. ex Boiss., Fl. Orient. Suppl. 154. 1888. TYPE: EGYPT: Al Bahr al Ahmar, Wadi Righbeh, 1887, Schweinfurth 228 (HOLOTYPE: G).

Pistacia khinjuk var. microphylla Boiss., Fl. Orient. Suppl. 154. 1888. TYPE: EGYPT: Al Bahr al Ahmar, upper Wadi Rigbeh, Schweinfurth 96 (HOLOTYPE: G; ISOTYPE: P).

Pistacia khinjuk var. macrocarpa Zohary, Palestine J. Bot. Jersulam Ser. 5:212. 1952. TYPE: IRAQ: Ninawa, Iskeftendivan, N of Shaaikh Adi, 1933, E. Guest 3666 (HOLOTYPE: K).

Trees, 2 - 5(-10) $\mathrm{m}$ high. Leaves deciduous, imparipinnate, $11-17.7 \mathrm{~cm}$ long, $8-15.5 \mathrm{~cm}$ wide, membraneceous; petiole angled or rounded; rachis not winged. Leaflets 1 - 9, 4.5 - $8.5 \mathrm{~cm}$ long, 1.5 - $4 \mathrm{~cm}$ wide, average ratio 2.3-1, opposite to subopposite, ovate to broadly ovate, acuminate, glabrous; terminal leaflet $2.9-8.5 \mathrm{~cm}$ long, 1.2 - $4 \mathrm{~cm}$ wide, often larger than laterals. Staminate panicles to $9 \mathrm{~cm}$ long, loosely branched, with large, white-pubescent bracts; flowers crimson. Pistillate panicles to $16 \mathrm{~cm}$ long, branched from base, stout, minutely pubescent to glabrous. Drupes red, to $8 \mathrm{~mm}$ in diameter.

Phylogeny.-The closest relative of $P$. vera [7].

Phenology.-Flowering March to May; fruiting April to October.

Habitat and ecology.-Rocky limestone slopes, sandy loam, and granite; 400 - $2400 \mathrm{~m}$.

Uses.-Afghanistan: "The nuts are roasted and eaten" (Aitchison 234, 606). "They make oil from the seeds for lighting" (Thesiger 1670). Iraq: "The kernel and the stone is eaten (the flesh of the fruit is bitter and cannot be eaten)" (Just 1643). Pakistan: "seeds edible" (Ghafoor \& Goodman 4586). Saudi Arabia: "red berries. edible" (Collenette 710).

Common names.-Gulungoor, Gwun, Khinjuk, Shurumma (Afghanistan), Ushgai Buzgai (Baluchistan) [10]. Iran: Baneh (Popov 51/203); Guan (Léonard 5889). Iraq: Butum, Gazwan (Kurdish), Habbat (Just 1643). Pakistan: Gwan (Ghafoor \& Goodman 4936); Ushgai (Pushtu) (Wace 3693).

Distribution.-Turkey south to Egypt and Saudi Arabia and east to Tajikistan, Afghanistan, Pakistan, and Nepal.

Specimens examined. AFGHANISTAN. Paktia: Kurrum [Kurram] Valley, 23 Nov 1878, J.E.T. Aitchison 234 (K); Kurrum [Kurram] Valley, Apr 1879, J.E.T. Aitchison s.n. (K); Kurrum [Kurram] Valley, 08 Sep 1880, J.E. T. Aitchison 606 (BM). Konar: Kargal Valley, $6000 \mathrm{ft}$, 01 Sep 1956, W. Thesiger 1670 (BM). Unknown region: Griffiths 1270 (K). EGYPT. Janûb Sîna': Sinai, 1930, A. Kaiser 693 (K); Wadi Thoneimin, near Wadi Isln, 30 mi S of St. Catherines Monastery, 1300 m, 11 Feb 1968, N. Tadmor S-74 (MO). IRAN. Jamal Basiz Mts., 7-9000 ft, 05 May 1951, G. Popov 51/203 (BM). Baluchestan: Zahedan, $19 \mathrm{mi} \mathrm{S}$ of Zaboli toward Sarbaz, $4400 \mathrm{ft}, 19$ Mar 1971, C. Grey-Wilson \& T.F. Hewer 216 (K). Kerman: Kuh-e Khabr, $10 \mathrm{~km}$ E of Khabr, $28^{\circ} 49^{\prime} \mathrm{N} 56^{\circ} 25^{\prime} \mathrm{E}$, 2400 m, 09 Jun 1977, Assadi et al. 1723 (E). Kermanshahah: Bisitun, $34^{\circ} 23^{\prime} \mathrm{N} 47^{\circ} 25^{\prime} \mathrm{E}, 1350$ - $1650 \mathrm{~m}, 23$ Jun 1963, M. Jacobs s.n. (K). Hormozgan: Quest du Jaz Murian, Route vers Bandar Abbas, $27^{\circ} 25^{\prime} \mathrm{N} 57^{\circ} 15^{\prime} \mathrm{E}, 530$ m, 28 Apr 1972, J. Léonard 5889 (K). Lurestan: May 1852, J. Olguin s.n. (BM); Sheshom, $32^{\circ} 45^{\prime} \mathrm{N} 48^{\circ} 15^{\prime} \mathrm{E}$, 500 - 800 m, 25 Apr 1963, M. Jacobs 6417 (E). Sastan: Harmuk (cult.), 27 Mar 1964, M.L. Grant 15336 (MO). IRAQ. Arbil: Rawandiz Gorge, 1500 ft, 20 Apr 1932, E. V. Just 2142 (K). As Sulaymanigah: Garai Goran-Tawilah-Haraman, 25 Jun 1973, Weinert \& Mousawi s.n. (F); Jarmo [E of Kirkuk], along wadi W of camp, $30 \mathrm{Apr}$ 1955, H. Helbaek 1272 (K). Dahûk: Bikhair Mt., near Zakhö (90 km NW of Mosul), 800 - 1200 m, 02 Jul 1957, A. Rawi 23063 (K). Nin: Tel Sinjar, 14 Nov 1962, F.A. Barkley \& S. Haddad 3889 (MO). Ninawa: Sheikh Adi, $\mathrm{N}$ of Mosul, $2750 \mathrm{ft}, 10$ Oct 1931, E.V. Just 1643 (K); near Ain Sifni, 13 Jun 1934, H. Field \& Y. Lazar 708 (F). 
JORDAN. Ma'an: Bada, Little Petra, 04 Jun 2004, M.G. AL-Saghir JOR24 (VPI); Bada, Little Petra, 04 Jun 2004, M.G. AL-Saghir JOR2 (VPI); Petra, 04 Jun 2004, M.G. AL-Saghir JOR26 (VPI); Petra, 04 Jun 2004, M.G. ALSaghir JOR 27 (VPI). NEPAL. Shimi, 28 $59^{\prime} \mathrm{N} 82^{\circ} 35^{\prime} \mathrm{E}$, 1300 m, 23 Apr 1974, J.F. Dobremez 2707 (BM). PAKISTAN. Balochistan: about $100 \mathrm{~km}$ from Bella toward Khuzdar, 07 May 1990, A. Ghafoor \& S.M. Goodman 4936 (F); between Khash and Iranshahr, 1500 - 1600 m, 16 - 17 May 1948, K.H. Rechinger \& F. Rechinger 4025 (MO); about $3 \mathrm{~km}$ from Kikin, between Shahrak and Turbat, 13 Mar 1990, A. Ghafoor \& S.M. Goodman 4586 (F); about 18 mi from Larma, 11 Jun 1970, O. Qaisn et al. 1447 (MO); Onza, between Zhob and Shinghar (cult.), 03 Jul 1988, T. Ali \& T. Ahmed 1063 (BM); Quetta, Loralei to Harnai, Torkhan Pass above Dilkuna, 1400 m, 14 May 1965, J. Lamond 1245 (E); Murdar Range, 31 Aug 1942, J. Sinclair 2144 (E); Wám Tangi Forest, 4000 ft, 05 Apr 1889, J.H. Lace 3693 (K). North-West Frontier Province: Waziristan, J.D. Hooker 254 (K). Punjab: Sufaid Koh, J.D. Hooker 138 (K). SAUDI ARABIA. Al Madinah: Harrat Rahah, $32 \mathrm{~km} \mathrm{SW}$ of Badiah, $5600 \mathrm{ft}, 27$ Apr 1995, I.S. Collenette 9368 (E). Hijaz: Jabal Harb, near Wadi Sawawin, 5000 ft, 03 May 1978, I.S. Collenette $710(\mathrm{~K})$. Tabuk: Jabal Dubbeagh, $85 \mathrm{~km} \mathrm{SW}$ of Tabuk, 4500 ft, 23 Apr 1983, I.S. Collenette 4380 (E, K); NW side of Jabal Dubbeagh, 5500 ft, 22 Mar 1978, I.S. Collenette 470 (K). TAJIKISTAN. Leninobad: near Buldzhuana, along Surkhob River, 11 Jul 1963, Khamilov s.n. (K). TURKEY. Mardin: Kasrik Bogazi [Gurpindar], 9 - 11 km from Gizre, 400 m, 07 May 1966, Davis 42652 (E, K). Siirt: Baykan to Siirt, 24 Jun 1954, Davis 22130 (BM); Botan Çay gorge, 19 km from Siirt, 1646 m, 19 May 1966, Davis 43285 (K).

\subsubsection{Pistacia terebinthus L., Sp. Pl. 2:1025. 1753}

Lentiscus terebinthus (L.) Kuntze, Revis. Gen. P1. 153. 1891. Type: Annotated "Terebinthus 4" (in Linnaeus' script). "Hispania 690" (in unknown script). (lectotype, selected here: LINN 1170.4).

Comments-Both Stearn (1957) [23] and Jarvis (2007) [24] emphasise the importance of the numbers that Linnaeus wrote on the sheets of many of the specimens in his herbarium for the typification of his names. "Such numbers almost always link these specimens with the corresponding account in the first edition of Species Plantarum" (Jarvis 2007: 43). Furthermore, "Stearn, from long study of the herbarium specimens and protologues over many years, reached the conclusion that the presence of such a number provided extremely strong evidence that the collection in question was in Linnaeus' possession by 1753" (Jarvis 2007:44). Pistacia terebinthus is species number 4 in Species Plantarum, This specimen is not the holotype, as it is not cited in Linnaeus' protologue.

Lentiscus vulgaris Garsault, Trait. P1. Anim, Med. 1: 63. 1767. TYPE: not designated.

Pistacia therebinthus Scop., Fl. Carniol. ed. 2. 2:262. 1771. nom. illeg. (based in part on $P$. terebinthus L. ICBN, Art. 52).

Pistacia crassifolia Salisb., Prodr. Stirp. Chap. Allerton 2:172. 1796. nom. illeg. (P. terebinthus L. is listed as a synonym; ICBN, Art. 52).

Pistacia palaestina Boiss., Diagn. Pl. Orient.Ser. 1, 9: 1. 1849. Pistacia terebinthus subsp. palaestina (Boiss.) Engl. in A. \& C. DC. Monogr. Phan. 4:290. 1883. TYPE: PALESTINE: Galilee, Boissier (LECTOTYPE: G).

Pistacia terebinthus var. angustifolia Lec. \& Lamot. ex Lamot., Prodr. Fl. Plat. Centr. 180. 1877. TYPE: not designated.

Pistacia terebinthina St.-Lag., Ann. Soc. Bot. Lyon 7: 132. 1880. nom. illeg. (based on P. terebinthus L.; ICBN, Art. 52).

Pistacia terebinthus var. vulgaris Engl. in A. \& C. DC. Monogr. Phan. 4:289. 1883. TYPE: not designated.

Pistacia terebinthus var. macrocarpa Zohary, Palestine J. Bot. Jerusalem Ser. 5:209. 1952. TYPE: ALGERIA:

Tissemsilt, Col de l'Oued Massin, near Teniet-el-Had, 1871, Chebert (HOLOTYPE: FI).

Pistacia terebinthus var. oxycarpa Zohary, Palestine J. Bot. Jerusalem Ser. 5:209. 1952. TYPE: TUNISIA: Jebel Ichkeut, 1887, Letourneux (HOLOTYPE: C).

Shrubs or small trees, 2-6(-12) $\mathrm{m}$ high. Leaves deciduous, impari- or paripinnate, $10-19 \mathrm{~cm}$ long, 6 - 19 $\mathrm{cm}$ wide, becoming coriaceous; petiole rounded, rarely flattened; rachis not winged. Leaflets (3-) 6 - 11, 3.5 - 8 $\mathrm{cm}$ long, $1-3.1 \mathrm{~cm}$ wide, average ratio $2.7-1$, opposite to subopposite, ovate to narrowly ovate, mucronate, minutely pubescent to glabrous; terminal leaflet if present 1 - $6 \mathrm{~cm}$ long, 5 - $20 \mathrm{~mm}$ wide, smaller than laterals. Staminate panicles to $8 \mathrm{~cm}$ long, diffusely branched, with large bracts bearing long white trichomes. Pistillate panicles $15(-23) \mathrm{cm}$ long, larger in fruit, stout, minutely puberulent to glabrous, large bracts with long white trichomes soon falling. Drupes reddish, ripening blue.

Phylogeny. $-P$. palaestina and $P$. terebinthus are closely related ( they are the same species) [7].

Phenology.-Flowering March to July; fruiting April to October.

Habitat and ecology.-Limestone, dolomite, schist; $225-1850 \mathrm{~m}$.

Common names.-Adharia (Arabic), Butum (Arabic), Ela (Hebrew), Scornabecco, Terebinte, Terebintha, Terebinto [7]. Cyprus-turpentine, Pistachier, Térébinthe, Terebinth, Turpentine-tree [22].

Distribution.-Native to the Mediterranean basin, from Spain to Turkey, Croatia, and Bosnia, and Morocco to Saudi Arabla, Jordan, and Syria. 
Specimens examined. ALGERIA. Ain Defla: below Ain N'Sour, Tipasa to Miliana, 900 - 1000 m, 30 May 1975, Davis 58367 (BM, E). Bejaïa: Cap Carbon, near Bejaïa, 250 m, 29 May 1971, Davis 52964 (BM). Djelfa: near Takesane Forest House, 45 - $50 \mathrm{~km}$ SW of Djelfa, 1400 m, 08 Jun 1971, Davis 53383 (BM, E). Sidi Bel Abbes: near Bossuet, 1300 m, 11 Sep 1929, A. Faure s.n. (K). Tiaret. Gueurtoafa, near Tiaret, Aug 1846, Romais s.n. (BM); Ras el Ain, Romais s.n. (BM). BOSNIAHERZEGOVINA. Szovito, 1848, G. Zohcabése 100 (K). CROATIA. Dalmatia, Aeschinger s.n. (K). DubrovnikNeretva: Narenta [Neretva], Kargl s.n. (F). Istria: Lavrana, 09 May 1898, M.F. Spencer S.n. (F); between Parenzo [Porez] and Revigno [Rovinj], 16 Aug 1922, W.B. Turrill 996 (K). Primorje-Gorski-Kotar: near Fiume [Rijeka], Noe 345 (K); 14 May 1869, A.M. Smith s.n. (K); 19 May 1870, coll. unknown (K); 29 Aug 1871, coll. unknown (K). Sibenik-Knin: N of Sibenik, 12 May 1969, R.K. Brummitt 6629 (K). CYPRUS. Castello Kantara, 18 Apr 1880, Sintenis \& Rigo 17 (K); Karaman, 300 m, 18 Jul 1994, DV 1451 (K); mountain above Kykko Monastery, 1150 m, 11 May, T.F. Hewer 4629 (K). FRANCE. Ain: Culoz, base of Colombier, 08 Jun and 02 Jul 1879, A. Déséglise 96 (BM). Alpes-Maritimes: Cipières Commune, 580 m, 07 Jun 1994, F.N. Hepper 9887 (K); Nice, Coteau du Vinaigrier, 04 May 1862, Canut 2 (F); Roquefort Notre Dame, 225 m, Taton 2012 (MO); Ardeche: 12 mi N of Pont St Esprit, 350 ft, 13 Sep 1953, P.A. Rawdon 46 (K). Gard: W of Rhône, opposite Avignon, 10 Jun 1952, J. Soustas 1247 (K); Vigan, 23 Apr to Jul 1859, G. Tuezkiewicz S.n. (F). Lot: Cénevieres, 07 Mar 1874, J.R. Bousquet s.n. (F); 08 Sep 1872, L. Giraudias s.n. (F); between Eleges and St. Denis prés Martel, Oct 1990, coll. unknown (K). Hautes Pyrénées: Gèdre, Rochers d'Agos, 05 Jul 1878, Bordère s.n. (F). Hérault: Grammont, 14 May 1888, Cinbract s.n. (F). Pyrénées Orientales: Sorède, 24 Apr to 20 Jun 1900, L. Conill s.n. (F). Unknown region: Pech de l'Aguel, 09 Jun 1888, Cinbract s.n. (F); L'Sauveur, 22 Jun 1886, Cinbract s.n. (F). GREECE. Agionoros: near Stavronikita [Athos Peninsula], $21 \mathrm{Apr}$ 1934, A.W. Hill et al. 2559 (K). Crete: Gorge de Aradaena, W of Chora Sphakion, 580 m, 10 May 1984, R. Deschamps 9018 (MO); La Caneé, Cheriso, 08 Jun 1914, $M$. Gandoger 4375 (MO); Lakki, S of Kania, 08 May 1984, R. Dechamps 9059 (MO); Melidosis, 04 Aug 1893, A. Baldacci 152 (K); Rethymo, Arkadi, 18 Jun 1914, M. Gandoger 5113 (MO). Dodekanisos: Rhodes, between Maritsa and Psinthos, 19 May 1983, K. Boratynska et al. 189 (K); Mt. Marmara, near Lardos, 13 May 1983, K. Boratynska et al. 145 (K). Drama: Bozdag, $10 \mathrm{mi} \mathrm{NW}$ of Drama, $500 \mathrm{ft}, 19$ May 1959, J.D.A. Stainton 7490 (K). Kefallonia: S Cephalonia, 16 Oct 1834, Schimper \& Wiest s.n. (K). ITALY. Toscana: Filettole, May 1861, coll. unknown (F); Pisan to Pietro and Padule, Apr 1857 and
May 1860, P. Savi s.n. (F). Trentino-Alto Adige: Riva, May 1872, Porta s.n. (K). Triesta: Trieste, 1826, Fleischer s.n. (K). Unknown region: near Benaeum, 230 500 m, 17 May 1865, Porta s.n. (F, K); Brix, near Benaeum, 2 - 500 m, 25 Apr 1869, Porta s.n. (K). JORDAN. Ajloun: Ajloun, 1029 m, 24 May 2004, M.G. AL-Saghir JOR05 (VPI); Ajloun, 1032 m, 24 May 2004, JOR07 (VPI); Ajloun City, 857 m, 24 May 2004, M.G. ALSaghir JOR11 (VPI); Ajloun City, 740 m, 29 May 2004, M.G. AL-Saghir JOR12 (VPI). Al Balqa': As-Salt, 830 m, 01 May 1911, F.S. Myers \& J.E. Dinsmore 52 (K). Irbid: Umm Qays, 275 m, 29 May 2004, M.G. AL-Saghir JOR02 (VPI). Jerash: Jerash, 1048 m, 29 May 2004, M.G. AL-Saghir JOR14a (VPI); 29 May 2004, M.G. ALSaghir JOR14b (VPI); Nyma, 1017 m, 24 May 2004, M.G. AL-Saghir JOR09 (VPI). Ma'an: Mt. Hor \& Petra, Nov 1883 - Feb 1884, H.C. Hart s.n. (BM). LEBANON. Al Janub: Barghoutié [Barghut] valley, near Saida [Sidon], 25 Aug 1857, Gaillardot 978 (BM, F). Beyrouth: Beirut, 23 May 1871, G.E. Post 261 (BM). Jabal Lubnan: Aley ['Aleih], 800 m, 19 Apr 1933, G. Samuelsson 1116 (MO); Wadi Sistrine above El Hermel, 3000 - 5000 ft, 16 Aug 1945, P.H. Davis 9853 (K). LIBYA. Tarabulus: Suk et Sebet, km 40 between Tripoli and Tarhunan, 300 m, 28 Apr 1963, Mazzocchi 1217 (K). MOROCCO. Beni-Bu-Yahi, Djebel Kerker, 850 - 1000 m, 14 Jun 1933, Sennen \& Mauricio 8727 (BM); Jebel Tisouka, above Xanen, 900 - 1000 m, 05 Jul 1975, Davis 54792 (BM); West Rif, Jbel Lakraa, 35 07'N $5^{\circ} 08^{\prime} \mathrm{W}$, 1450 - 1560 m, 11 Jun 1995, A. Boratynski \& A. Romo R-8599/6 (BM); Zarkat, 1250 m, 16 Jul 1927, Font Quer 374 (BM); Zerhoun, Jebel Kemfoud, 550 m, 10 May 1929, E. Jahandiez 179 (BM). Beni Mellel: S of El Ksiba, 01 Aug 1966, R.M. \& A.M. Harley 855 (BM); between Zaouia Ahanesal and Tilougguite, 1850 m, 15 Jul 1973, Davis 55241 (BM). Moyen Atlas: Azrou, 1500 m, 08 Aug 1924, E. Jahandiez 906 (BM); near Azrou, 25 Jun 1919, H. Lynes 123 (BM). PALESTINE. Deires Sheikh, 20 Aug 1945, E. Hardy s.n. (BM); Jerusalem, 30 May 1902 F. Meyers 28 (F); 12 Apr and 09 Jul 1904, F.S. Meyers 2028 (F); 22 Apr 1905, J.E. Dinsmore 4028 (F); Upper Galilee, Wadi Qarn, 30 May 1926, A. Eig \& M. Zohary s.n. (MO); Mt. Carmel, 1863-1864, B.T. Lowe s.n. (BM); Wadi Fallah (Carmel), 25 Mar 1942, P.H. Davis 4180 (K); Wadi Shomaza (Carmel), 18 Aug 1942, P.H. Davis 4928 (K). SAUDI ARABIA. Grande Lavone, 3000 ft, 04 Apr 1982, J. Grainger 447 (E). Al Madinah: Jabal Radhua, N of Yenbo [Yanbu 'al Bahr], $6500 \mathrm{ft}, 10$ Oct 1981, I.S. Collenette 2896 (K). SPAIN. Granada: Sierra Nevada, Guejar Sierra, 1000 - 3500 ft. 28 Aug 1846 \& Mar 1847, H.M. Willkomm 1312 (K). Jaén: Jabalcuz, 750 m, 01 Jul 1986, C. Fernández S.n. (K). León: Villafranca del Vierzo, 06 Jul - 15 Jul 1860, J. Lange s.n. (K). Mallorca: Gorg Blau, $19 \mathrm{~km}$ from Soller toward Pol- 
lensa, 500 m, 13 Jul 1969, L.F. Ferguson \& I.K. Ferguson 2472 (MO). Oviedo: Río Salla gorge, $18 \mathrm{~km} \mathrm{~S}$ of Cangas de Onis, $29 \mathrm{Jul}$ 1985, P. Frost-Olsen 7471 (MO). SYRIA. Al Ladiquiah: below Kessab, $2000-2500 \mathrm{ft}$, 11 May 1945, F.H. Norris s.n. (BM). Al Qunaytirah: Banias [Banaiyas], 05 Apr 1877, G.E. Post 426 (BM). Halab: mountains between Aleppo \& Antioch, P. Russell s.n. (BM). TUNESIA. Bizenta: Parc National d'Iehkeul, 1978-1979, J.M. Fay 1250 (K). TURKEY. Adana: Feke, 750 m, 14 Apr 1957, Davis \& Hedge 26699 (E). Amasya: Amasia [Amasya], Mar - Apr 1889, Bornmüller 209 (BM). Antalya: Gebiz, Pisidie, 1100 m, 27 Jul 1949, P.H. Davis 15779 (E); $50 \mathrm{~km}$ from Kas toward Finike, 350 m, 30 May 1988, S. Zmarzty \& S. Atkins 166 (K); Termessos, 900 m, 36 $59^{\circ} \mathrm{N} 30^{\circ} 30^{\prime} \mathrm{E}, 25$ Sep 1989, M.P. Frankis 89.25 (E). Asia Minor: Hafis Pasha, 800 m, 12 Apr 1936, T.A. Tengwall 282 (K). Gümüshane: near Torul, 10 Sep 1993, M. Pitman \& A. Wickham 238 (K). Hatay: $30 \mathrm{~km} \mathrm{~S}$ of Antioch, $800 \mathrm{~m}, 08$ Jun 1938, J.E. Dinsmore 20392 (K); Rabat, 100 m, 05 May 1965, M.J.E. Coode \& B.M.G. Jones 486 (K). Kütahya: Gediz, 850 m, 07 Jul 1962, Davis \& Coode 36958 (E). Manisa: S of Demiroi toward Salihli, 600 m, 20 Jun 1965, M.J.E. Coode \& B.M.G. Jones 2769 (K). Mugla: Hisarönü to Turgut, 50 - 100 m, 15 Apr 1965, Davis 41140 (E); Kalkan, 30 m, 30 Mar 1956, Davis \& O. Polunin 25503 (K). Sakarya: W of Adapazari [Sakapari], 50 m, 08 Aug 1962, Davis \& Coode 39181 (E). Sinop: above Gerze, 300 m, 07 Sep 1954, Davis \& O. Polunin 25010 (BM, E). Tokat: Tokat-Niksar, 04 Sep 1954, Davis \& O. Polunin 24881 (K). Trabzon: Sumela Gorge, S of Trabzon, 03 Jun 1977, C.R. Lancaster s.n. (BM). Vilayet: near Magmur Dag, 400 m, 01 Jun 1963, C. Tobey 205 (E).

\subsubsection{Pistacia vera L., Sp. Pl. 2:1025. 1753}

Lentiscus vera (L.) Kuntze, Revis. Gen. Pl. 153. 1891. Type: illustration, "Pistacia." in J. Bauhin, Historia plantarum, 1(1): 275. 1650. (lectotype, selected here).

Comments-Both Stearn (1957) and Jarvis (2007) strongly make the point that when choosing a lectotype for a Linnean name it is best to choose a specimen rather than an illustration. However, in this case it not possible to do so. None of the specimens of $P$. vera in Linnaeus' herbarium can be used to typify this name. No. 1170.1 (not annotated by Linnaeus) appears to have been added after 1753. No. 1170.2 (annotated "2 narbonensis Kh" [Linnaeus' student Martin Kahler] in Linnaeus' script) is a type of $P$. narbonensis. No. 1170.3 (annotated "Pistacia 3 " in Linnaeus' script) is from George Clifford's herbarium (BM), but Hortus Cliffortianus (Linnaeus 1738) is not cited in his protologue; it is also annotated "an a $\mathrm{P}$ Terebinthus diversa? Non es vera" by J.E. Smith and indeed is $P$. terebinthus.

There is a specimen in Joachim Burser's herbarium at
Uppsala (Herb. Burser XXII: 60. UPS) that Linnaeus identified as $P$. vera [26,27]. Burser's herbarium was arranged according to C. Bauhin (1623) (see Stearn 1957: 116-118). Linnaeus consulted Burser's herbarium at the University of Uppsala and entered his identifications of the species into his own copy of a later edition of $\mathrm{C}$. Bauhin (1671) [28]. Linnaeus cited Bauhin in numerous species' protologues in Species Plantarum, including that for $P$. vera. Unfortunately, the specimen is unidentifiable: "Junge Zweige, vielleicht von Pistacia vera L." (Juel 1936:145).

Three of the references cited by Linnaeus in his protologue contain illustrations. That of J. Bauhin (1650) is the most complete and recognizable as $P$. vera, with leaves, infructescences, and fruits. Lobel (1576:413) is poorer, with leaves and fruits. Tournefort (1700, 3:380; "Cotinus"), with flowers and fruits, is unidentifiable.

Pistacia trifolia L., Sp. Pl. 2:1025. 1753. TYPE: not designated.

Pistacia narbonensis L., Sp. Pl. 2:1025. 1753. pro parte TYPE: see above discussion.

Pistacia nigricans Crantz, Inst. Rei Herb. 1:184. 1766. TYPE: not designated.

Pistacia officinarum Aiton, Hortus Kew. 3:398. 1789. nom. illeg. (P. trifolia $\mathrm{L}$. is listed as a synonym; ICBN, Art. 52).

Pistacia variifolia Salisb., Prodr. Stirp. Chap. Allerton 171. 1796. nom. illeg. (P. trifolia L. and P. officinarum Aiton are listed as synonyms).

Pistacia reticulata Willd., Sp. Pl. ed. 4. 4(2):751. 1806. nom. illeg. (P. narbonensis $\mathrm{L}$. is listed as a synonym).

Pistacia macrophylla Pers., Syn. Pl. 2(2):615. 1806. nom. illeg. (P. trifolia $\mathrm{L}$. is listed as a synonym).

Pistacia badghysi K. P. Popov, Izv. Akad. Nauk Turkmen. SSR, Biol. Nauk 5:5. 1978. TYPE: TURKMENISTAN: Mary Welayaty: Kushkam mountains, ShorSafel, E of Morgunovskiy, 28 Aug 1977. K. Popov (holotype: LE; isotype: WIR).

Shrubs or trees, $1-5 \mathrm{~m}$ high, young branches minutely pubescent. Leaves deciduous, imparipinnate, 10.2 - 17 $\mathrm{cm}$ long, $8.4-16 \mathrm{~cm}$ wide, coriaceous, clustered at ends of branches; petiole flattened; rachis flattened but not winged, minutely pubescent. Leaflets $3-5,4-8 \mathrm{~cm}$ long, 3 - $5.5 \mathrm{~cm}$ wide, average ratio $1.6-1$, lower often subopposite, ovate to broadly ovate, obtuse or mucronulate, midveins and margins minutely pubescent; terminal leaf-let $6-10 \mathrm{~cm}$ long, $3-8 \mathrm{~cm}$ wide, often larger than laterals. Staminate panicles to $5 \mathrm{~cm}$ long, forming globose clusters. Pistillate panicles to $5.5 \mathrm{~cm}$ long and $2 \mathrm{~cm}$ in diameter in flower, cylindrical, minutely pubescent. Drupes reddish to pale green, to $18 \mathrm{~mm}$ long and $10 \mathrm{~mm}$ wide.

Phylogeny. - P. vera is sister to P. khinjuk [7].

Phenology.-Flowering May, June, August, October; 
fruiting April to June.

Common names.-Pistachio, Pistache, Fistashka (Russian), Pista (Persian), Pistacchio (Italian), Pistacier (French), Fustuq Halabi (Arabic), Botne (Hebrew) [10]. Green almond, Pistachier cultivé, Pistazie, Pistazienbaum, Pisutachio, Alfóncigo, Pistachero [22].

Distribution.-Wild $P$. vera is native to the eastern Mediterranean from Turkey to Palestine and Syria, and east to Central Asia (Turkmenistan, Uzbekistan, Afghanistan, Tajikistan, and Kyrgyzstan.

Specimens examined. AFGHANISTAN. Badakhshan: Faisabad to Meshed [Mashad], Warduj Valley, $30 \mathrm{mi} \mathrm{W}$ of Faisabad, $4500 \mathrm{ft}$, 01 Jun 1964, P. Furse 6443 (K). Badghis: $150 \mathrm{mi}$ NE of Herat, pass NE of Qala-i-Naw, $4500 \mathrm{ft}, 28$ Jun 1971, C. Grey-Wilson \& T.F. Hewer 1275 (K). Baghlan: Kataghan, Paigah, Kotal, between Pul-i-Khumri [Pol-e Khomri], $35^{\circ} 55^{\prime} \mathrm{N} 68^{\circ} 45^{\prime}$ E, and Haibak, $36^{\circ} 15^{\prime} \mathrm{N}, 68^{\circ} 03^{\prime} \mathrm{E}, 1500 \mathrm{~m}, 1967$, K.H. Rechinger 33910 (K). Unknown region: 14 May 1885, J.E.T. Aitchison s.n. (K); 03 May 1885, coll. unknown 390? (K). CYPRUS. Castello Kantara, 18 Apr 1880, Sintenis \& Rigo s.n. (BM); Limassol, 50 ft (cult.), 20 Apr 1938, J. Michalides 360 (K). KAZAKHSTAN. Vostochnyy Kzakhstan: Tumkubassk, Kara-Taun [Karatan], upper Konbulan Valley near Uvala Ridge, 22 Aug 1939, A.V. \& V.A. Yarmolenko 112 (MO). Yuzhnyy: [Turkestan] Alatan. Dschil Aryk (Roamschlucht), Sep 1876, A. Regel s.n. (BM). PALESTINE. Jerusalem (cult.), Oct 1908 \& 23 Apr 1909, J.E. Dinsmore 21942 (F). SYRIA. Dimashq: Aïn Tinéh, NE of Damascus. 29 Aug 1856, Gaillardot 979 (F). Halah: near Aleppo [Halah], Frere s.n. (BM). TAJIKISTAN: near Dushanbe, 08 Jun 1960, Romahnof s.n. (E); 25 May 1960, Shtsbahkobah s.n. (MO); 930 m, 24 Jun 1967, G. Nyehpdn s.n. (K, MO). TURKEY. Sanliurfa: Cümcüme, near Samsat, $37^{\circ} 27^{\prime} \mathrm{N} 38^{\circ} 27^{\prime} \mathrm{E}$, ca 400 m (cult.), 1983-1984, N.F. Miller 886 (K). TURKIMENISTAN. Ahal Welayaty: Ashkhabad [Ashgabut], 500 - 600 m, 13 May 1975, V.V. Neketen \& E.A. Evahnov s.n. (BM, K); Badchys [Reserve, Eriolanduz, Marey District], $30 \mathrm{Jul}$ and 10 Aug 1910, N. Samokisch \& N. Androssov 7428 (K, MO); SW Kopet-Dag Mts, PalvanZau [Kora-Kok region], 1975, N. Belianina \& G. Proskuriakova 165 (MO); W Kopet Dag, W part of valley of Tersakan, $38^{\circ} 23^{\prime} \mathrm{N} 55^{\circ} 57^{\prime} \mathrm{E}$, 30 Jun 001, D. Kurbanov 776 (MO). USA. California: Chico, USDA Plant Introduction Station, (cult.), 10 Aug 1960, USDA s.n. (F, 2 colls). UZBEKISTAN. Buchara [Bukhara] or Ticket, May 1883, Regel s.n. (BM).

\subsection{Pistacia L. sect. Lentiscella Zohary, Palestine J. Bot. Jerusalem Ser. 5:194. 1952}

Pro parte Eu Lentiscus Zohary, Palestine J. Bot. Jerusalem Ser. 5:194. 1952. pro parte Lentiscus Parfitt \&
Badnes, Proc. Natl. Acad. Sci. USA. 94:7987-7992. 1997. Type: Pistacia mexicana.

Pistacia section Eu Lentiscus Zohary is an illegitmate name because the epithet in the name of a subdivision of a genus is not to be formed from the name of the genus to which it belongs by adding the prefix $E u$ - (ICBN, Art. 21. 3). Pistacia section Lentiscus Parfitt \& Badnes was published without a Latin diagnosis.

Small trees or shrubs; leaves evergreen, paripinnate or imparipinnate; leaflets 6-32; rachis narrowly winged.

Includes the following species: P. lentiscus L., P. mexicana HBK, and $P$. weinmannifolia Poiss. ex Franch.

\subsubsection{Pistacia lentiscus L., Sp. Pl. 2:1026. 1753}

Terebinthus lentiscus (L.) Moench, Methodus 345. 1794. Type: Herb. Linn. No. 1170.8 (LINN) (Siddiqi in Jafri \& El-Gadi (ed.), Fl. Libya 52:3. 1978) (lectotype: LINN 1170.8).

\subsubsection{Pistacia lentiscus subsp. lentiscus}

Pistacia messiliensis Mill., Gard. Dict. ed. 8. n. 6. 1768. Pistacia lentiscus var. massiliensis (Mill.) Duhamel, Traité Arbr. Arbust. 4:72, 1809. Lentiscus massiliensis (Mill.) Fourr., Ann. Soc. Linn. Lyon. Sér. 2, 17:195. 1869. TYPE: not designated (Figure 2).

Pistacia gummifera Salisb., Prodr. Stirp. Chap. Allerton 2:172. 1796. nom. illeg. (P. lentiscus is listed as a synonym; ICBN, Art. 52).

Pistacia lentiscus var. chia Duhamel, Traité Arbr. Arbust. 4:72, [t. 8. delete] 1809. TYPE: not designated.

Pistacia lentiscus var. angustifolia DC., Prodr. 2:65. 1825. nom. illeg. (P. messiliensis Mill. is listed as a synonym).

Pistacia lentiscus var. latifolia Coss., Notes Pl. Crit. Ser. 1, 54. 1850. TYPE: not designated.

Pistacia brevifolia Gand., Dec. Pl. Nov. 1:44. 1875.

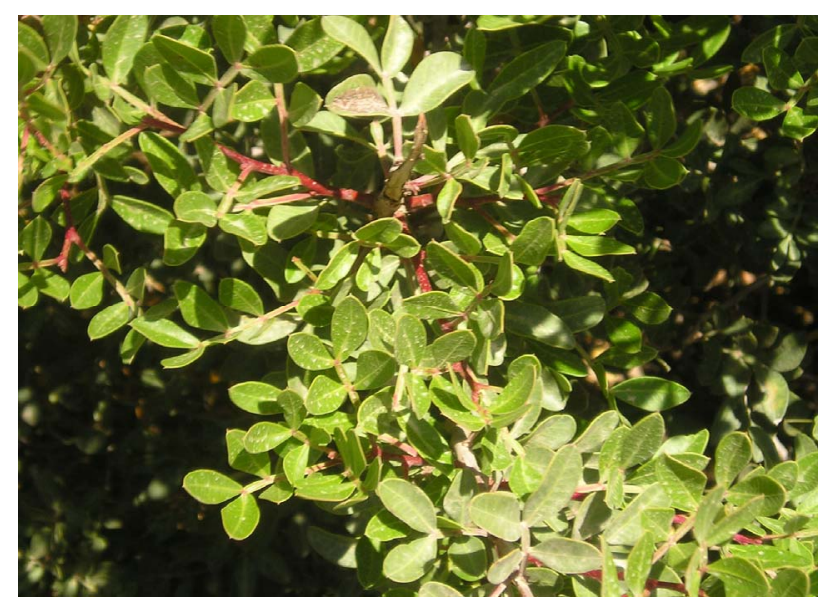

Figure 2. Pistacia lentiscus subsp. lentiscus shrub. This picture shows the sub species subcoriaceous leaves. This species is located in Jerash, Jordan. Scale bar 1:1000. 
TYPE: not designated.

Pistacia subfalcata Gand., Dec. Pl. Nov. 1:44. 1875.

TYPE: not designated.

Pistacia multiflora Gand., Dec. Pl. Nov. 1:44. 1875. TYPE: ITALY: Pisa: Monte Pisano, near Pisa, P. Savi (LY).

Pisacia lentisca St.-Lag, Ann. Soc. Bot. Lyon, 7:132. 1880. nom. illeg. (based on P. lentiscus L.; ICBN, Art. 52).

Pistacia lentiscus f. leptophylla Albo ex Fiori, Nuov. Fl. Italia 2:117. 1923. TYPE: not designated.

Shrubs or rarely small trees, $1-5 \mathrm{~m}$ high. Leaves evergreen, usually paripinnate, $2-7.5 \mathrm{~cm}$ long, $3.4-10 \mathrm{~cm}$ wide, subcoriaceous; petiole flattened; rachis narrowly winged. Leaflets 4 - 10 (-12), $1.6-4.5 \mathrm{~cm}$ long, 4 - 17 $\mathrm{mm}$ wide, average ratio $2.8-1$, more or less opposite to subopposite, sessile, ovate to narrowly ovate or elliptical, rarely obovate, mucronate or rarely mucronulate, glabrous, margin thick, undersurface noticeably lighter than upper surface; terminal leaflet rarely present, $1.4-2.1 \mathrm{~cm}$ long, 4 - $9 \mathrm{~mm}$ wide, same size or smaller than laterals. Staminate panicles and racemes axillary, $4-5 \mathrm{~cm}$ long, greenish, densely-flowered and spike-like when young. Pistillate panicles and racemes axillary, clustered with leaves, to $4 \mathrm{~cm}$ long, greenish, spike-like when young. Drupes yellow-green turning crimson, $4-5 \mathrm{~mm}$ in diameter.

Phylogeny.-P. lentiscus subsp. lentiscus and P. lentiscus subsp. emarginata (P. aethiopica) form a close pair [7].

Phenology.-Flowering December to September; fruiting April and July to November.

Habitat and ecology.-Scrub, matorral, open oak forest; calcareous gravelly soil, rocky limestone, loamy sand; $0-2000 \mathrm{~m}$.

Common names.-Akind, Droug, Elath, Hamastiq, Lentischio, Lentisque, Ochinos, Saris, Sondro [7]. Almecegueiralentisco, Arbre au mastic, Chios mastictree, lentisque, Mastixbaum [22]. Canary Islands: Almacigo (s. coll. 56). Palestine: Balm (American Colony 815); Mastic (American Colony 815, Dinsmore 4815). Spain: Lentisco (Schinini \& Sánchez García 30609); Mata (Martindale 54, 158). Tunesia: Battum (Keith 166).

Distribution.-From the Canary Islands across southern Europe from Portugal to Greece and Turkey, across North Africa from Morocco to Egypt and the Middle East.

Specimens examined. ALBANIA. Vadica to Valona [Vlora], 01 Aug 1892, A. Baldacci 189 (K). ALGERIA. Algiers: Alger [Algiers], May 1849, P. Jamin s.n. (F); Algiers, Apr 1857, coll. unknown (K); Forêt du Bainem, $13 \mathrm{~km} \mathrm{~W}$ of Algiers, $37^{\circ} \mathrm{N} 03^{\circ} \mathrm{E}, 1000 \mathrm{ft}, 14 \mathrm{Jan} 1978, R$. P. Croston s.n. (MO); Hussein-Dey, near Alger [Algiers], 14 Apr 1860, C. Romain 1963 (F, 2 colls.). Boumerdes:
El Kaddous, 06 Apr 1851, P. Jamin 141 (K, 2 colls.). Oran: Oran to Santa-Cruz, 31 Aug 1930, A. Faure s.n. (MO); Forêt Moulay-Ismaël, near Tlélat, 27 Jul 1930, A. Faure s.n. (MO). CANARY ISLANDS. Fuerteventura: Rio Palmas, 06 Apr 1859, R.T. Lowe 212 (BM); Virgenes de la Peña, coll. unknown 56 (BM). Gran Canaria: Findley s.n. (K); R.T. Lowe 54 (BM); El Dragonál, Mar 1846, E. Bourgeau 406 (K); Monte Lentiscal, 26 Apr 1894, R.P. Murray s.n. (K); 400 m, 01 Feb 1969, G. Kunkel 12438 (BM, MO); Tafira, Apr 1897, A.C. Cook 523 (F, MO); Tafira Alta, 400 m, 28 Mar 1969, D. Bramwell 1096 (E). CROATIA. Cres: Monte Ossero [Oser], 21 May 1887, O. Stapf s.n. (K). Dalmatia: Biograd, 23 Apr 1962, F. Höpflinger s.n. (F); Molunat, $15 \mathrm{~km} \mathrm{~W}$ of Hercegnovi [Herzegovina], 0 - 30 m, 19 Aug 1977, P. Frost-Olsen 1414 (MO). Dubrovacko-Neretvanska Zupanija: near Gravosa [Gruz], Lapad Peninsula, 04 Aug 1925, Turrill W.B. 1027 (K). CYPRUS. near top of Bofarento, 29 Jul 1898, coll. unknown (BM); Karaman, 250 m, 8 Jul 1994, DV 14501 (K); W of Karmakiti, 09 Apr 1967, Landfenton 134 (K); Kyrenia, 0 m, 20 Mar 1956, J. C. Atherton 1198 (K). EGYPT. Aswan: Abu Simbel (cult.), Feb 1904, R. Muschler s.n. (MO). Port Said: Port Said, Feb 1904, R. Muschler s.n. (MO). FRANCE. Alpes Maritaimes: Cannes, May 1900, K.H. Marguerite s.n. (F); Cap d'Antibes, Nov 1876, Dyer s.n. (K); Coteau du Sinaigrié, near Nice, 04 May 1862, Canul 842 (F, 2 colls.); Frèjus, 19 Apr 1861, E. Bourgeau 109 (F); Menton, Cap Martin, 09 Feb 1869, coll. unknown (K); Therebontauae, coll. unknown (F). Bouches-du Rhône: Aixen-Provance, May 1854, E.A. Willmott s.n. (K). Hérault: Pic St. Loup, $10 \mathrm{~km}$ E of St. Martin de Londres, $43^{\circ} 46^{\prime} \mathrm{N}$ 034'ㄹ, 400 m, 06 Oct 1993, B. Summers et al. 6511 (MO). Orles: Cousolation, Penchinat, May 1851, Billot 1640 (F). Var: Brignoles, 10 Nov 1928, coll. unknown (F); Le Luc (8ar), Apr 1869, Faure s.n. (F); Toulon-surmer, Apr 1857, J. Parseval s.n. (F). GREECE. Chalkidiki: head of Cassandra Gulf, 12 Apr 1934, A.W. Hill et al. 2608 (K, 2 colls.). Crete: Akrotiri, 13 Apr 1914, M. Gandoger 3317, (MO); Kisamos, Gonia, 23 Mar 1914, M. Gandoger 4709 (MO); Sitia, Toplon, 25 Apr 1914, M. Gandoger 357 (MO). Dodekanisos: Rhodos, Istrios, Kaiserswald, Jun 1855, F.W. Areschoug s.n. (F). East Macedonia \& Thrace: Kizil Ada, 200 ft, 18 Apr 1937, H.G. Tedd 1958 (K). Evvoia: Euboea, Sotir Hagios Monestary, near Kymi, 05 Sep 1969, K.H. Rechinger 38178 (MO). Kerkyra: Corfu [Kerkyra], Castello Hotel, $300 \mathrm{~m}$, 23 Apr 1971, W.R. Presl 369 (K). Kyklades: Melos [Milos], 300 m, 17 Apr 1976, A.M. Young 393 (K). Notio Aigaio: Sifnos, Profitus Illias, 800 ft, 22 Aug 1963, Royal Liberty School 55 (K). Thespratia: Epiros, Parga, S of Igoumenitsa, 21 Aug 1964, B. Verdcourt 4129 (K). Voreio Aigaio: Chios, Aucher-Eloy 927 (BM). ITALY. Foggia: Lesina, Apr 1843, R.C. Alexander s.n. (K). Im- 
peria: Ospedalletti, between San Remo and Bordighera, Apr 1931, Metcalfe s.n. (K, 2 colls.). Napoli: Capri, 06 Apr 1892, M.F. Spencer s.n. (F); Naples, Kings Park Portici, 1854, coll. unknown (K); Pozzuoli, P. Savi s.n. (F). Pisa: Monte Pisano, 1846, Maggio s.n. (F); Pisa, Botanic Garden (cult.), 1863, H.H. Babcock s.n. (F). Reggio di Calabria: Scilla, 21 Jun 1965, R.K. Brummitt et al. 5256 (K). Sardinia: Aranci Bay, Apr 1907, J.S. Gamble 28269 (K, 2 colls.). Savona: Capo di Noli, coll. unknown (F). Unknown region: (cult.), Mar 1907, $R$. Park 3 (F). JORDAN. Jerash. Jerash, Akhasheiba, 503 m, 29 May 2004, M.G. AL-Saghir JOR13a, JOR13b (VPI). LEBANON. Jabal Lubnan: Beirut, $500 \mathrm{ft}$, Apr 1945, F.H. Norris s.n. (BM). LIBYA. Al Fatih: Farzuga, near Barce [Al Marj], 16 Aug 1957, K. Prichard CYR/57/ 31 (BM). Al Jabal Al Akhdar: Tocra Pass, between Benghazi and Derna [Darnah], 19 Aug 1952, O. Brittan 9 (BM); Wadi Kouf, W of Beida [Al Bayda'], 300 m, 28 Mar 1970, Davis 50201 (E). Darnah: Derna [Darnah], 23 Jul 1908, T.F.W. Gregory s.n. (BM, 2 colls.); Ras el Hilal, 500 m, 28 Feb 1958, H.G. Keith 626 (K). MOROCCO. Agadez: between Cap Ghir and Agadir, $26 \mathrm{Mar}$ 1972, D. Bramwell et al. 254 (MO); Immouzer Valley, N of Agadir, 28 Aug 1972, G. Bramwell et al. 280 (K, MO). Agadir: between Cap Ghiv and Agadir, 26 Mar 1972, D. Bramwell et al. 254 (K). Al Hoceima: Beni Bu-Frah [Boufrah], 50 m, 05 Jun 1927, Font Quer 373 (BM); between Ketama and El Hoceima, 920 m, 16 May 1972, J. C.M. Alexander \& F.K. Kupicha 235 (BM). Ben Silmane: Bouznika, 60 m, 23 Apr 1979, J. Lewalle 9019 (BM, 2 colls.). Beni Mellal: Afourer to Bin-el-Ouidane, 1300 m, 13 Jul 1973, Davis 55163 (BM, MO). Essaouira: Djebel Hadid, near Mogadore [Essauoira], Apr - May 1871, J.D. Hooker s.n. (K); Mogador [Essaouira], 25 Apr 1859, R.J. Lowe 32 (BM); Feb 1934, A.W. Trethewy 120 (K). Fes: Bir-Tamtam, 12 Nov 1965, A. Taton 1669 (BM). Kenitra: $10 \mathrm{~km} \mathrm{SW}$ of Kenitra toward Mehdia Plage, $34^{\circ} 13^{\prime} \mathrm{N} 6^{\circ} 41^{\prime} \mathrm{W}, 30 \mathrm{~m}, 27$ Oct 1994, S.L. Jury et al. 15170 (K). Khemisset: Imouzzer-des-Ida-Outanane to Oulma, 500 m, 21 Mar 1969, P. \& J. Davis 48503 (E). Marrakech: $5 \mathrm{~km}$ from Asni toward Imlil, $31^{\circ} 12^{\prime} \mathrm{N}$ $07^{\circ} 58^{\prime} \mathrm{W}, 1285 \mathrm{~m}, 19 \mathrm{Jul}$ 1989, M. Ait Lafkih et al. 683 (BM); Imigdal, $5 \mathrm{~km} \mathrm{~N}$ of Tizi-n-Test, $31^{\circ} 01^{\prime} \mathrm{N} 08^{\circ} 09^{\prime} \mathrm{W}$, 2000 m, 06 Apr 1974, Miller et al. 778 (BM; MO, 2 colls.); N of Tiz-n-Test Pass toward Marrakech, 31 Mar 1972, D. Bramwell et al. 515 (K, MO). Meknes: Jebel Zerhoun, above Moulay Idriss, $34^{\circ} 02^{\prime} \mathrm{N} 05^{\circ} 30^{\prime} \mathrm{W}, 1080 \mathrm{~m}$, 23 Oct 1993, S.L. Jury et al. 12611 (BM); $9 \mathrm{~km} \mathrm{E} \mathrm{of}$ Moulay Idriss toward Nzala-des-Beni-Ammar, $34^{\circ} 04^{\prime} \mathrm{N}$ $05^{\circ} 27^{\prime} \mathrm{W}, 600$ m,04 Jun 1994, S.L. Jury et al 14951 (BM). Nader: Hemis de Tensaman, 04 Jun 1931, Sennen \& Mauricio (BM). Oujda: near Debdon, 900 - 1000 m, 03 Apr 1928, E. Wilczek et al. 170 (MO). Skhirat-Temara: Rabat toward Zaen,. 200 m, 23 Jun 1992, J. Lewalle
13877 (MO); Temara (Rabat), Oued Iquem, 100 m, 13 Mar 1981, J. Lewalle 9710 (BM, MO); 50 m, 18 Mar 1984, coll. unknown 10893? (BM). Tanger: Grottes d'Hercules, NW of Tanga, $35^{\circ} 46^{\prime} \mathrm{N} 05^{\circ} 56^{\prime} \mathrm{W}, 10 \mathrm{~m}, 16$ Oct 1993, S.L. Jury et al. 12194 (BM, K); Oued Dliane, E of Tanga toward Sebta, $35^{\circ} 50^{\prime} \mathrm{N} 05^{\circ} 39^{\prime} \mathrm{W}, 5 \mathrm{~m}, 17$ Oct 1993, S.L. Jury et al. 12243 (BM); Tangier and Tetuan, Apr 1871, J.D. Hooker s.n. (K). Tetouan: Djebel Habibi, 1910-1911, M. Gandoger s.n. (MO); El Fandek Pass, W of Tetouan toward Zarache, 24 Mar 1972, D. Bramwell et al. 42 (K, MO); Ouriba, Apr - May 1871, J.D. Hooker s.n. (K); Reraja, Jun 1872, Rein \& von Fritsh 449 (K). PALESTINE. Ahuya Sir Henry Samuel, Sep 1946, J.L. Gilbert 36 (K); Bet. Masir, 02 Sep 1903, F. Meyers 815 (F); Caiffa [Haifa], 15 Oct 1860, J.D. Hooker \& D. Hanbury s.n. (K); Jerusalem, 03 May 1907, J.E. Dinsmore 4815 (F); Kiryath-Anavim, near Jerusalem, 16 Oct 1933, A. Eig et al. 260 (E); Lower Galilee, top of Mt. Tabor, 31 Mar 1954, D. Jaffe \& C. Shenkar s.n. (MO); Mt. Carmel, 200 m, 28 Mar 1911, F.S. Meyers \& J.E. Dinsmore 7126 (F); Valley of Ajalon, American Colony 815 (MO); Wadi Fallah, Carmel, 16 Feb 1942, P.H. Davis 3947 (K). PORTUGAL. Alrantes, Oct 1850, J. Ball s.n. (F). Coimbra: between Cabo Mondego and Sierra de Boa, $9 \mathrm{~km}$ from Viagem, 209 m, 16 Sep 1967, R. Wilczek 2464 (MO). SPAIN. Andalucia: Sierra de la Cruz, 1000 m, 24 Jul 1925, coll. unknown (K). Cadiz: Cadiz, 1826, Pignant s.n. (K). Jaén: Pozoalcón, Cerro Miguel, 900 - 1000 m, 09 Jun 1987, E. Villanueva et al. 1042EVG (F); Sierra Morena, Andújar, 15 May 1953, A. Rodríguz s.n. (K). Majorca: Pollensa, $300 \mathrm{ft}, 22$ Oct 1934, G. Martindale 54 (K); 200 - 300 ft, 27 Jan 1936, coll. unknown 158? (K). Murcia: Venta de los Pinos, 24 May 1996, A. Schinini \& S. Sánchez García 30609 (F). Valencia: $5 \mathrm{~km}$ from Finestrat, $38^{\circ} 35^{\prime} \mathrm{N} 00^{\circ} 12^{\prime} \mathrm{W}, 08$ Apr 1993, C.C.H. Jongkind \& R.M.J. Nieuwenhuis 772 (MO); $5 \mathrm{~km}$ from La Nucia and $8 \mathrm{~km}$ from Benidorm, $38^{\circ} 35^{\prime} \mathrm{N} 00^{\circ} 08^{\prime} \mathrm{W}, 10$ Apr 1993, C.C.H. Jongkind \& R.M.J. Nieuwenhuis 795 (MO). SYRIA. Sep - Oct 1860, J.D. Hooker \& D. Hanbury s.n. (F); 1863-1864, B.T. Lowne s.n. (BM, K). TUNISIA. Jendouba: Forêt de l'Oued Zeen, $20 \mathrm{~km}$ SE of Tabarka [Tabarqah], 600 m, 14 Jun 1965, J.W.A. Jansen 564 (MO); Forêt du Feidja, $20 \mathrm{~km} \mathrm{~W}$ of Ghardimaou, 16 Apr 1965, J.W.A. Jansen 166 (MO); Kroumirie, between Les Chenes and Ain Draham, 400 m, 07 May 1984, Davis 70025 (E). Le Kef: between Nehenh and Le Kef, $1000 \mathrm{ft}, 15$ Mar 1925, W.P. Lowe \& D.A. Bannerman 155 (BM). Siliana: $14 \mathrm{~km}$ from Maktar [Makthar] toward Kairouan, 820 m, 03 May 1975, Davis \& Lamond 57146 (BM, E). Zaghouan: Zriba, on road to Enfidaville, km 72 from Tunis, 18 Sep 1957, H.G. Keith 166 (K). TURKEY. Adana: Misis, near Adana, $200 \mathrm{ft}, 11 \mathrm{Apr}$ 1934, E.K. Balls B729 (BM). Antalya: [Okulu, 13 Feb 1966, coll. unknown 8515 (MO)]; Antalya to Alauya jolu, 
20 km from Alanyaya, 23 Feb 1966, A. Baytop 8515 (E); Kiremi-thaneler, $15 \mathrm{~km} \mathrm{SW}$ of Antalya, $40 \mathrm{~m}, 07 \mathrm{Apr}$ 1959, E. Hennipman et al. 136 (K). Mersin: between Tarsus and Ulas, 200 m, 05 Apr 1957, Davis \& Hedge 26417 (BM).

\subsubsection{Pistacia lentiscus L. subsp. emarginata (Engl.) AL-Saghir, Comb. Nov.}

Basionym: Pistacia lentiscus. var. emarginata Engl. in A. \& C. DC., Monogr. Phan. 286. 1883. Type: SOMALIA: Meid Ahlgeb, 1000 m, 1875, J.M. Hildebrandt 1531. (holotype: BM!).

Pistacia aethiopica Kokwaro in Kokwaro \& Gillett, Kew Bull. 34:755. 1980. TYPE: SOMALIA: Meid Ahlgeb, 1875, J. M. Hildebrandt 1531 (HOLOTYPE: K; ISOTYPE: BM!).

Trees or shrubs, 2 - $15 \mathrm{~m}$ high. Leaves evergreen, paripinnate, $4-10 \mathrm{~cm}$ long, $3.8-8.4 \mathrm{~cm}$ wide, coriaceous; petiole flattened of angled; rachis winged. Leaflets $6-16$, 1.9 - $4.2 \mathrm{~cm}$ long, 6 - $16 \mathrm{~mm}$ wide, average ratio $2.6-1$, subopposite to alternate, ovate to narrowly ovate, rarely obovate, emarginate, glabrous. Staminate panicles to 2.5 $\mathrm{cm}$ long, dark red, branched from base, spike-like when young; flowers reddish. Pistillate panicles to $5.5 \mathrm{~cm}$ long in fruit, branched from base; flowers reddish. Drupes reddish, ca. $5 \mathrm{~mm}$ in diameter.

Phylogeny. - P. lentiscus subsp. emarginata (P. aethiopica) and P. lentiscus form a close pair [7].

Phenology.-Flowering January, April, May, and October; fruiting January, February, May, July, and November.

Habitat and ecology.-1200 - $2700 \mathrm{~m}$.

Uses.-Somalia: "gum called Gubelli" (Glover \& Gilliland 550).

Common names.-Hamar, Heis, Sisaye, Ulaaso (Zohary, 1952). Ethiopia: Borana (Ash 2447). Kenya (Masai): Oldaangudwa (Rammell 3488). Somalia: Hais (Glover \& Gilliland 550).

Distribution.-East Africa (Ethiopia, Somalia, Kenya, Uganda, Tanzania).

Specimens examined. ETHIOPIA. Oromia: Neghelle [Negale], $05^{\circ} 20^{\prime} \mathrm{N} 39^{\circ} 40^{\prime} \mathrm{E}, 4850 \mathrm{ft}, 22$ Jan 1954, H.F. Mooney 5607 (K); 27 mi SE of Neghelle, 13 Apr 1974, J.W. Ash 2447 (K, MO); $20 \mathrm{~km} \mathrm{~S}$ of Neghelle toward Melka Guba, 1500 m, 18 May 1982, I. Friis et al. 3119 (K); $34 \mathrm{~km}$ on Negele-Filtu road, $05^{\circ} 15^{\prime} \mathrm{N} 39^{\circ} 47^{\prime} \mathrm{E}, 1650$ m, 09 May 1980, M. Thulin et al. 3450 (K). KENYA. Eastern: Mt. Kulal, Gatab, 1600 m, 21 Nov 1978, F.N. Hepper \& P.M.L. Jaeger 6972 (K, MO). Rift Valley: J.C. Rammell 3488 (K); Chyulu hills, $02^{\circ} 34^{\prime} \mathrm{S} 37^{\circ} 48^{\prime} \mathrm{E}$, 20 Oct 1969, J.B. Gillett \& F. Kariuki 18816 (K, MO); Kilima Kiu, 1926, Mrs. P. Decie s.n. (BM); 9 mi SE of Narok, 6200 ft, 23 Jul 1957, C.G. Trapnell 2339 (K). Tere: native reserve, $6200 \mathrm{ft}, 1914$, Ms. P. Decie s.n. (BM). Un- known region: E.R. Napier 2325 (K). SOMALIA. Sanaag: Erigavo, Daloh Forest, 26 Jan 1945, P.E. Glover \& H.B. Gilliland 550 (BM); Obda doho, Daloh Forest, 07 Jul 1945, P.E. Glover \& H.B. Gilliland 1129 (BM); Surud [Shimbiris], 2350 m, 17 Jan 1973, J.J. Lavranos \& Horwood 10359 (E); Surud Forest Reserve, 20 - 24 km NW of Erigavo, 17 Jan 1973, P.R.O. Bally \& R. Melville 16001 (K, MO). Woqooyi Galbeed: Gan Libah, Golis range [Gar Golis], $5400 \mathrm{ft} .16 \mathrm{Jul}$ 1945, P.E. Glover \& H. B. Gilliland 1162 (BM, K); Libah Mele Mt., above Biyu Suldan [Buja Soldan], 5200 - 5950 ft, 03 Dec 1932, J.B. Gillett 4697 (K). Unknown region: Meid Ahlgeb, 1100 m, Apr 1875, J.M. Hildebrandt 1531 (BM, holotype). TANZANIA. Arusha: Gendabi, Mt Hanang, $7500 \mathrm{ft}, 10$ Feb 1946, P.J. Greenway 7690 (K); M'bulu, 17 May 1954, L. Matalu 3124 (K). UGANDA. Karamoja: Timu forest, 5000 - $6000 \mathrm{ft}$, Apr 1960, J. Wilson 1017 (K).

\subsubsection{Pistacia mexicana Humb., Bonpl. \& Kunth, Nov.} Gen. Sp. 7:22, t. 608.1824

Lentiscus mexicana (Humb., Bonpl., \& Kunth) Kuntze, Revis. Gen. Pl. 153. 1891. Type: MEXICO. Guerrero: Chilpancingo, 1825, Bonpland (BM).

Pistacia texana Swingle, J. Arnold. Arbor. 2:107. 1920. TYPE: TEXAS: Valverde Co.: Rio Grande valley near mouth of Pecos River, near Hinojose Spring, 18 Mar 1911, S.C. Mason (HOLOTYPE: US; ISOTYPE: AA).

Trees or shrubs, 2 - $15 \mathrm{~m}$ high. Leaves evergreen, clustered towards ends of branches, usually imparipinnate, $5.1-15.1 \mathrm{~cm}$ long, $1.8-5.3 \mathrm{~cm}$ wide, membranaceous; petiole flattened; rachis narrowly winged. Leaflets 10 $30,1-2.5 \mathrm{~cm}$ long, $3-10 \mathrm{~mm}$ wide, average ratio $2.7-1$, narrowly ovate, slightly falcate, mucronate, glabrous; terminal leaflet $5-25 \mathrm{~mm}$ long, $3-10 \mathrm{~mm}$ wide, same size or smaller than laterals. Staminate panicles to $4 \mathrm{~cm}$ long, reddish to yellowish, clustered at branch tips with and below expanding leaves, densely flowered, pubescent. Pistillate panicles to $10(-17) \mathrm{cm}$ long in fruit, branches spreading, clustered towards ends of branches below leaves; flowers red. Drupes red, purple, blue, or black, 4 $5 \mathrm{~mm}$ in diameter, pendulous.

Phylogeny.-P. mexicana and P. texana form a close pair (AL-Saghir, 2010).

Phenology.-Flowering February, March, May, August; fruiting April to August.

Habitat and ecology.-Hillsides, deciduous scrub, dry forest, open pine-oak woods; limestone mountains, calcareous soil over limestone, rocky or gravelly areas; 550 $-2200 \mathrm{~m}$.

Common names. -Copall [7]. Mexican Pistachio [22]. Guatemala: Copalillo (Steyermark 32975). Mexico: Copál (Hinton et al. 10258); Lantrisco (Wilson M-11); Tezelpom (Tzeltal, Ton 5589).

Distribution.-Native to southern Texas, Mexico, Gu- 
atemala, and Honduras.

Specimens examined. GUATEMALA. Baja Verapaz: near Santa Rosa, 1500 m, 04 Apr 1941, P.C. Standley 91089 (F); N of Santa Rosa, 30 Mar 1939, P.C. Standley 69749 (F). Chiquimula: Quetzaltepec, El Guatalón, 900 m, 17 May 1999, M. Vélez 99.7046 (BM). Guatemala: Estancia Grande, 600 m, 08 Dec 1938, P.C. Standley 59171 (F); near Fiscal, 1080 - 1140 m, 12 Dec 1938, P.C. Standley 59573 (F); 1100 m, 18 Dec 1940, 80412 (F). Huehuetenango: near Aguacatán, near spring of San Juan, 1600 m, 27 Dec 1940, P.C. Standley 81370 (F); Ochuptaj, Río Nentón, $15^{\circ} 48^{\prime} \mathrm{N} 91^{\circ} 45^{\prime} \mathrm{W}, 800 \mathrm{~m}, 30$ Nov 1993, J.J. Castillo \& J.A. Castillo 2081 (F); Puente El Aguilar, E of San Rafael Pétzal, 1730 m, 29 Dec 1940, P.C. Standley 81411 (F); above San Sebastián H., 2000 - 2200m, 13 Aug 1942, J.A. Steyermark 50518 (F). Jalapa: between Jalapa and San Pedro Pinula, 1400 - 1800 m, 12 Nov 1940, P.C. Standley 77021 (F); W of San Pedro Pinula, 1000 m, 12 Nov 1940, P.C. Standley 77124 (F); near Zapote, $1 \mathrm{mi}$ NE of San Pedro Pinula, 1500 m, 09 Dec 1939, J.A. Steyermark 32975 (F). Quiché: 1942, J.I. Aguilar 846, 1157(F). HONDURAS. Comayagua: Rancho Grande, San Luis, 4000ft, 22 May 1932, J.B. Edwards P-269 (F). Francisco Morazán: between Cantarranas and San Juancito, 722 m, 24 Mar 1957, A. Molina R. 7789 (F); Pinares, $20 \mathrm{~km}$ W of Cedros, 900 - $1000 \mathrm{~m}, 28$ - 30 May 1976, C. Nelson \& E. Vargas 3481 (MO); Puente Colorado, N of Tegucigalpa, 900 - 1000 m, 07 Dec 1949, P.C. Standley 24932 (F); Quebrada El Chimbo, SW of Tegucigalpa, 1200 m, 17 Mar 1957, A. Molina R. 7760 (F); Quebrada la Orejona, 750 m, 24 May 1985, G. Rodríguez M. s.n. (MO); Quebrada de Suyapa, near Suyapa, 1100 1200 m, Sep - Dec 1948, P.C. Standley 12938 (F); Quebrada Suyapa, Aldea de Suyapa, NE of Tegucigalpa, 1200 m, 07 Oct 1948, A. Molina R. 1206 (F, MO); 1100 m, 10 Aug 1949, coll. unknown 2551?, (F, MO); between Quebrada Tarán and Valle de Angeles, 1300 m, 03 Aug 1962, A. Molina R. 11094 (F); near Suyapa, 1200 m, 25 Nov - 14 Dec 1946, P.C. Standley \& L.O. Williams 1421 (F, 2 colls.); 13 Dec 1946, L.O. Williams \& A. Molina R. 1421 (F, 2 colls.); Tegucigalpa, 07 May 1975, M. Erazo 86 (MO). Olancho: Campo Abierto, 960 m, 08 May 1984, R. Ramos 109 (MO); 2 km NE San Francisco de la Paz toward Gualaco, 700 m, 04 Apr 1983, McCarter \& Hughes 4 (MO). MEXICO. Chiapas: S of Amatenango del Valle, 5900 ft, 14 Mar 1967, A.S. Ton 2144 (F, MO); Arroyo Jeshab, 900 m, 05 Mar 1983, A.M. Ton 5589 (MO); 800 m, 20 Apr 1983, coll. unknown 5874? (MO); junction of Mexican Highway 190 and road to Bochil and Simjovel, $3400 \mathrm{ft}, 10 \mathrm{Apr}$ 1965, D.E. Breedlove 9624 (F); above El Chorreadero, 800m, 16 Apr 1972, D.E. Breedlove $24568(\mathrm{~F}) ; 4 \mathrm{mi} \mathrm{W}$ of Comitán, $5750 \mathrm{ft}, 26$ Apr 1949, M.C. Carlson 1994 (F); 10 km NE of Las Margaritas toward La Soledad, 1700 m, 17 Feb 1973, D.
E. Breedlove 33391 (MO); Reserva del Ocote, road from Bajada del Macho, to rancho La Cruz, 08 May 1983, J.I. Calzada et al. 10098 (F); Trapichito, 1350 m, 02 Jun 1945, E. Matuda 15660 (F); $3 \mathrm{~km} \mathrm{~N}$ of Tuxtla Gutiérrez toward El Sumidero, $1800 \mathrm{ft}, 07$ Apr 1965, D.E. Breedlove $9567(\mathrm{~F}) ; 1 \mathrm{~km}$ SE of Tzimol toward Uninajob, $16^{\circ} 08^{\prime} \mathrm{N} 92^{\circ} 11^{\prime} \mathrm{W}, 1110 \mathrm{~m}, 24 \mathrm{Feb} 1988$, A. Reyes García et al. 338 (BM); $5 \mathrm{~km} \mathrm{SW}$ of Tzimol, $1150 \mathrm{~m}, 04$ May 1988, A. Reyes García 483 (BM); 80 km SE of Villa las Rosas toward Tzimol, 800 m, 22 Apr 1987, E. Martínez \& A. Reyes 20230 (BM). Coahuila: Cañon de la Gavia, $\mathrm{S}$ of Rancho de la Gavia, $26^{\circ} 18^{\prime}-20^{\prime} \mathrm{N} 101^{\circ} 15^{\prime}-18^{\prime} \mathrm{W}$, 1250 - 2200 m, 02 - 03 Aug 1973, M.C. Johnston et al. 12001 (MO); N end of Cañon de la Hacienda, Sierra de la Madera, $27^{\circ} 06^{\prime} \mathrm{N} 102^{\circ} 25^{\prime} \mathrm{W}, 1500 \mathrm{~m}, 11$ May $1973, M$. C. Johnston et al. 10985 (MO); Cañon de la Madera, 4 $\mathrm{km}$ E of Rancho Buena Vista, Sierra de los Guajes, 07 Sep 1941, R.M. Stewart 1495 (F); Cañon de Milagro, E side of Sierra de los Guajes, $12 \mathrm{~km} \mathrm{~W}$ of Hacienda de la Encantada, 10 - 16 Sep 1941, R.M. Stewart 1509 (F); Mina El Aguirreno, Sierra de la Paila, $26^{\circ} 06^{\prime} \mathrm{N} 101^{\circ} 36^{\prime} \mathrm{W}, 1550$ m, 06 Jul 1973, M.C. Johnston et al. 11718 (F, MO); Monclova, 06 Aug 1939, E.G. Marsh 1982 (F); Muzquiz, 1935, E. Marsh 91 (F); Yerda spring, 07 Jul 1936, coll. unknown 358? (F); near El Puerto de San Lázero, 16 Jun 1936, F.L. Wynd \& C.H. Mueller 105 (K, MO); 1 km N of Rancho de la Gavia, Sierra de la Gavia, $26^{\circ} 20^{\prime} \mathrm{N}$ $101^{\circ} 14^{\prime} \mathrm{W}, 1200 \mathrm{~m}, 18 \mathrm{Mar} 1973$, M.C. Johnston et al. 10278 (F, MO); $5 \mathrm{~km}$ SE of Rancho Cerro de la Madera, Sierra de la Madera, $27^{\circ} 07^{\prime} \mathrm{N} 102^{\circ} 23^{\prime} \mathrm{W}, 1250 \mathrm{~m}, 20$ Sep 1972, F. Chiang et al. 9388 (MO); about $2 \mathrm{~km} \mathrm{SW}$ of Rancho San Miguel, N side of Serranías del Burro, $29^{\circ} 12^{\prime} \mathrm{N} 101^{\circ} 31^{\prime} \mathrm{W}, 575 \mathrm{~m}, 02$ Jun 1972, F. Chiang et al. 7502B (UT); Saltillo, 1898, E. Palmer 140 (BM, F, MO); Sierra del Carmen, $178 \mathrm{~km}$ from Múzquiz, $28^{\circ} 47^{\prime} \mathrm{N}$ $102^{\circ} 30^{\prime} \mathrm{W}, 1200 \mathrm{~m}, 20$ Aug 1994, M.A. Carranza et al. 2107 (MO); $4 \mathrm{~km} \mathrm{E}$ of San Francisco, 27 $30^{\circ} \mathrm{N} 102^{\circ} 33^{\prime} \mathrm{W}$, 1450 m, 10 Jun 1972, F. Chiang et al. 7605 (MO); Sierra de la Paila, N side just below Mina El San Luis, Aug 1878, C.C. Parry 98 (BM, K); Sierra Pata Galana, Mar 1905, C.A. Purpus 1117 (F). Guanajuato: $10 \mathrm{~km} \mathrm{~N}$ of Atarjea toward Los Cocos, 1100 m, 17 Apr 1989, E. Ventura \& E. López 6664 (MO); Rancho Beltran, $10 \mathrm{~km}$ S of Xichú, 1600 m, 15 Jun 1989, E. Ventura \& E. López 6782 (MO). Guerrero: Chichihualco, $11 \mathrm{~km} \mathrm{~W}$ of Xochipala, toward Filo de Caballo, 1640 m, E. Martínez S. 701 ( 2 colls.); 12 Apr 1982, coll. unknown 714? (F); 10.3 mi W of Chilpancingo toward Omiltemi, 1800 m, 27 - 28 Jul 1968, W. Anderson \& C. Anderson 4916 (MO); San Antonio, 900 m, 06 Jan 1937, Hinton et al. 10258 (F, MO). Hidalgo: $7 \mathrm{~km} \mathrm{~N}$ of Atotonilco el Grande, $1900 \mathrm{~m}$, 07 May 1981, R. Hernández M. et al. 6016 (MO); Barranca de Venados, Metzquititlan, 1800 m, 06 Aug 1979, R. Hernández M. 3615 (MO); Jacala, 4500 ft, 13 Jul 
1939, V.H. Chase 7445 (F, MO); $1 \mathrm{~km} \mathrm{~N}$ of Jacala, 08 Jun 1987, A. Garcia M. \& J. Miller 3192 (F, MO); near Lagunilla, Cerro Santa María, 2200 m, 03 Feb 1971, Rzedowski 28059 (F); near Pedrerito, 28 Apr 1981, D.H. Lorence \& D.L. Venable 3341 (MO). Jalisco: near El Salto del Río Jiquilpan, 28 Jun 1981, E.J. Lott \& J.A. Magallanes 387 (MO); Cerro Viejo, above Santa María Cuyutlán, 1850 m, 22 Jun 1991, T.S. Cochrane \& M. Cházaro B. 12617 (MO). Michoacán: Coalcoman, 1260 m, 09 May 1938, G.B. Hinton et al. 12135 (K); Zitacuaro to La Florida, 21 Jun 1938, G.B. Hinton et al. 11983 (MO). Nuevo Leon: below Alamar, about $15 \mathrm{mi}$ SW of Galeana, 45 - 5000 ft, 30 May 1934, C.H \& M.T. Mueller 630 (F); Diente Canyon, near Monterrey, Jul 1933, C.H. \& M.T. Mueller 333 (F); $8 \mathrm{~km}$ SE of Galeana, toward Linares, 1700 m, 24 Mar 1970, J. Rzedowski 27177 (F); Huajuco Cañon, $35 \mathrm{mi} \mathrm{S}$ of Monterrey, $3500 \mathrm{ft}, 29 \mathrm{Mar}$ 1954, A.F. Wilson M-11 (F); Lampazos, 21 Jun 1937, M. T. Edwards 286 (F); Montemorelos, Las Adjuntas, 2518' N $100^{\circ} 08^{\prime} \mathrm{W}, 750 \mathrm{~m}, 05$ Sep 1992, T.F. Patterson et al. 7098 (UT); Monterrey, Pastora, 550 m, Jul 1911, G. Arsène \& Abbon 6332 (MO); Rancho Las Adjuntas, Cañon Marisio Arriba, 24 Jun 1935, C.H. Mueller 2040 (BM, F, MO); Sierra Madre near Monterrey, Jun 1888, C. G. Pringle 1930 (F); Monterrey, 1835, C.G. Pringle s.n. (F). Oaxaca: Barranca Ceniza, NNW of El Parian, $17^{\circ}$ $25^{\prime} \mathrm{N} 97^{\circ} \mathrm{W}, 1500-1600 \mathrm{~m}, 30$ May 1992, A. Salinas T. et al. 6906 (MO); Cañada de Carrizalillo, Cerro Verde, 1600 - 1820 m, 17 Jul 1984, P. Tenorio L. et al. 6976 (F); Cerro El Ramón, 1 km W of El Rodeo, 2200 m, 08 Jul 1986, A. García M. et al. 2421 (MO); between Cuicatlán and Telixtlahuaca, 1800 m, 04 May 1978, E. Hernández $X .16746$ (F); Hierve el Agua, SW of San Lorenzo Albarradas, $16^{\circ} 52^{\prime} \mathrm{N} 96^{\circ} 17^{\prime} \mathrm{W}, 1760 \mathrm{~m}, 05$ Feb 1986, R.E. Gereau et al. 2038 (MO); Hierve el Agua, E of Mitla, $16^{\circ} 52^{\prime} \mathrm{N} 96^{\circ} 17^{\prime} \mathrm{W}, 1730 \mathrm{~m}, 23$ Apr 1989, S.C. Caran 5 (UT); 8 km E of Ixtlán de Juárez, 03 Apr 1981, R.C. Trigos \& D. Lorence 635 (BM, MO); Las Sedas, 1900 m, 19 May 1907, C. Conzatti 1802 (F); Río El Molino, 4.5 km N of Ixtlan, 2000 m, 27 May 1983, R. Cedillo T. \& D. Lorence 2327 (MO); San Juan Coatlan, 16 $6^{\circ} 5^{\prime} \mathrm{N} 96^{\circ} 53^{\prime} \mathrm{W}$, 1600 m, 18 Apr 1990, A. Campos V. et al. 3018 (F); San Miguel del Río, 1400 m, 18 Aug 1979, F. Ventura A. 16367 (MO); $2.5 \mathrm{~km} \mathrm{~W}$ of Oaxaca-Puerto Escondido highway toward San Sebastián de las Grutas, 1480 m, 16 May 1982, R. Torres C. et al. 452 (MO); $3 \mathrm{~km} \mathrm{~N}$ of Santos Reyes Tepejillo, $17^{\circ} 27^{\prime} \mathrm{N} 97^{\circ} 56^{\prime} \mathrm{W}, 1510 \mathrm{~m}, 19 \mathrm{Apr}$ 1996, J.I. Calzada 20923 (MO); Sierra de San Felipe del Agua, 24 May 1984, A. Saynes V. 11 (MO); Telixtlahuaca, "Parián-Las Sedas", 17²3'N 97'W, 1500 - 1625 m, 29 May 1992, A. Salinas T. et al. 6882 (MO); Tonaltepec, 1900 m, 26 Mar 1980, F. Gonzalez M. et al. 843 (F). Puebla: Agua Socoya, Barranca Agua Palenque, $18^{\circ} 13^{\prime} \mathrm{N}$ $97^{\circ} 24^{\prime} \mathrm{W}, 2070$ m, 14 Mar 2001, P. Tenorio L. 19998
(MO); Cañada de Mamacla, $12 \mathrm{~km} \mathrm{~W}$ of Molcaxac, 04 May 1982, F. González Medrano et al. 12380 (MO); Cerro Mezize, N of San Luis Atolotitlan, 2100 m, $10 \mathrm{Apr}$ 1984, P. Tenorio L. \& C. Romero de T. 5771 (F); Colonia San Miguel, $6 \mathrm{~km} \mathrm{SW}$ of Acatepec, 23 Mar 1980, F. Gonzales M. et al. (MO); Majada Salea, SW of San Simón, 1870 m, 24 Jun 1983, P. Tenorio L. \& C. Romero de T. 3957 (MO); Tehuacan, May 1912, C.A. Purpus 5848 (F); near Tehuacan, 5500 ft, 01 Aug 1901, C.G. Pringle 8553 (E, F, K); W of Tehuacán, 02 Aug 1901, J.N. Rose \& R. Hay $5858(\mathrm{~K}) ; 5.5 \mathrm{~km}$ from Zamarilla toward Magdalena y Clavijero, $18^{\circ} 28^{\prime} \mathrm{N} 97^{\circ} 46^{\prime} \mathrm{W}, 2025$ m, J.I. Calzada. 24053 (K). Queretaro: Río Moctezuma, $2.5 \mathrm{~km} \mathrm{~S}$ of Casa de Máquinas, Cadereyta, $1120 \mathrm{~m}, 28$ Apr 2000, S. Zamudio et al. 11387 (UT); San Luis Potosí: 6 km S of Acatepec, 23 Mar 1980, F. Gonzalez et al. F-734 (MO); Buena Vista, Nov 1911, C.A. Purpus 4889 (F); Minas de San Rafael, May 1911, C.G. Purpus 5362 (F); $8 \mathrm{mi}$ E of San Francisco, $22^{\circ} 03^{\prime} \mathrm{N} \mathrm{100}{ }^{\circ} 32^{\prime} \mathrm{W}$, 5500 ft, 05 Jun 1979, W. Hess \& L. Byrne 4643 (F). Tamaulipas: Cerro de los Armadillos, near San José, 10 Jul 1930, H.H. Bartlett 10206 (F); road to Cerro el Diente de San Carlos, 700 m, 03 Jun 1986, M. Yanez 805 (MO); $10 \mathrm{~km} \mathrm{~S}$ of Padron y Juarez toward Guerrero, 1100 m, 23 Mar 1985, M. Yanez 57 (MO); La Tamaulipeca, near San Miguel, 29 Jul 1930, H.H. Bartlett 10700 (F); near Victoria, 320 m, 01 Feb - 09 Apr 1907, E. Palmer 204 (F, K); between Victoria and Jaumave Valley, 31 May 1898, E.W. Nelson 4445 (F). Veracruz: $4 \mathrm{~km}$ from Acultzingo toward Orizaba, $2000 \mathrm{~m}, 28 \mathrm{Jul}$ 1971, Nevling \& Gomez-Pompa 2222 (F); Cerro de Achichuca above Tuzamapan-Huatusco highway, $19^{\circ} 23^{\prime} \mathrm{N} 96^{\circ}$ 49'W, 600 m, 09 May 1981, A. Vovides \& I. Calzada 665 (F). Unknown region: 1791, T. Haenke 1007, 1199, 1693 (F). USA. Texas: Val Verde Co., Hidden Trail Canyon, NW of Rio Grande about 8 mi below mouth of Pecos River, 18 Feb 1977, A.M. Powell 3055 (UT).

\subsubsection{Pistacia weinmannifolia J. Poiss. ex Franch., Bull. Soc. Bot. France 33:467. 1887}

Type: CHINA: "Yun-nan, circa Tapin-tze; fl. mart.; fr. jun. (Delav[ay]. n. 562 [P])."

Pistacia coccinea Collett \& Hemsl., J. Linn. Soc. Bot. 28:36. 1890. TYPE: MYANMAR: Shan. Shan Hills, 1888, Collett 649 (HOLOTYPE: K).

Pistacia malayana M.R. Hend., Gard. Bull. Straits Settlem. 7:97, t. 19. 1933. Pistacia weinmannifolia var. malayana (M.R. Hend.) Zohary, Palestine J. Bot. Jerusalem Ser. 5:202. 1952. TYPE: MALAYSIA: Perak, Gunong Runto, Lenggong, 1930, Henderson 23,831 (K).

Trees or shrubs, $1-15 \mathrm{~m}$ high. Leaves evergreen, paripinnate, $5-10.2 \mathrm{~cm}$ long, $2-6.4 \mathrm{~cm}$ wide, membranaceous; petiole flattened or angled; rachis narrowly winged. Leaflets $(4-6) 12-20,1-3.2(-9) \mathrm{cm}$ long, 5 - 
16(-37) mm wide, average ratio 2.6-1, alternate to subopposite, ovate to narrowly ovate, rarely obovate, glabrous, margin revolute. Staminate panicles to $4 \mathrm{~cm}$ long, spikelike when young, branches short; flowers bright pink. Pistil- late panicles to $10 \mathrm{~cm}$ long, clustered among terminal leaves, minutely pubescent; flowers red. Drupes bright green, red when mature, ovoid, to $7 \mathrm{~mm}$ long.

Phylogeny. $-P$. weinmannifolia is sister to P. Mexicana [7].

Phenology.-Flowering November to May, July; fruiting December, May to July.

Habitat and ecology.-Limestone outcrops; 480 - 2500 $\mathrm{m}$.

Uses.-leaves used for making incense (Henry 11913).

Common names.—China: Chin hsiang (Henry 11913).

Distribution.-Southwestern China, Myanmar, Vietnam, and Malaysia.

Specimens examined. CHINA. Guangxi. $1.5 \mathrm{~km} \mathrm{~W}$ of Hongdong, NW of Huanjiang, 960 m, 07 Mar 1997, P.J. Cribb et al. 433 (K). Guizhou: Po Gang, S of Xingyi, 1100 m, 13 Mar 1996, Z.H. Tsi et al. 52 (K). Sichuan: between Hualinpin and Chinchihsien, $1600 \mathrm{~m}, 29$ Jun 1934, H. Smith 10275 (MO); Nin-yüan-fu, 13 May 1922, H. Smith 1762 (MO). Xizang Zizhigu [Tibet]: Li Liching, Tsékeu, 17 Apr 1891, R.P. Soulie s.n. (K); Tsé-Kou, 17 Mar 1912, Monbeig 62 (E, K). Yunnan: NW, 1907, Monbeig s.n. (K); Baihualing, E side of Gaoligong Shan, 1460 m, coll. unknown 13953 (F); Chuxiong Xian, near Longtang, $159 \mathrm{~km} \mathrm{~W}$ of Kunming, $25^{\circ} 04^{\prime} \mathrm{N} 101^{\circ} 47^{\prime} \mathrm{E}$, 1820 m, 25 Jul 1984, Sino-American Botanical Expedition 1251 (E); Cao Xi Temple, Anning, $42 \mathrm{~km} \mathrm{~S}$ of Kunming, 2025 m, 29 Apr 1981, Sino-British Expedition to Cangshan K127 (E, K); Lao-Koui-Chan, 2500 m, Apr 1923, E.E. Maire 4 (K); Lijiang Hillside, 07 Jul 199, S.Y. Hu \& Y.C. Kong Y1179 (K, MO); Liekiang Valley, $27^{\circ} 30^{\prime} \mathrm{N}, 9000$ - $10000 \mathrm{ft}$, Jun 1913, G. Forrest 10153 (E); Lunan, A. Henry 9600A (BM); Machang, near Kieonyapin, 20 Feb 1909, F. Ducloux 1244 (E); coll. unknown 6236? (K); Mengsa, $4500 \mathrm{ft}$, A. Henry 9600, 9600B (K); Shonggui, 2100 m, 01 Jul 1996, Y. Lou 43 (K); Szemao, $8000 \mathrm{ft}, 05$ May, A. Henry 11913 (BM). Unknown region: $2000 \mathrm{~m}, 14$ May 1988, C.D. Chu 1218 (MO); Western China, dry warm valleys, $3000 \mathrm{ft}$, Jul 1903, E.H. Wilson 3367 (BM, K). MALAYSIA. Selangor: Bukit Takun, Kanching, 500 ft, 03 Nov 1937, M Nur 34398 (BM). MYANMAR. Tenasserin: Molodaung Ridge, 480 m, 21 Jan 1927, R.N. Parker 2457 (K). VIETNAM.

Cao Bang: near Ban Lung and Lung Phuc, about $50 \mathrm{~km}$ E of Cao Bang, $22^{\circ} 46^{\prime} \mathrm{N} 106^{\circ} 44^{\prime} \mathrm{E}, 650-700 \mathrm{~m}, 25 \mathrm{Nov}$ 1998, L. Averyanov et al. 661 (MO); about $39 \mathrm{~km} \mathrm{SSE} \mathrm{of}$ Cao Bang, 22 21'N 106 $27^{\circ} \mathrm{E}, 600 \mathrm{~m}, 03$ Dec 1998, Averyanov et al. 839 (MO); near Lung Hoai, about 43 $\mathrm{km} \mathrm{E}$ of Cao Bang, $22^{\circ} 43^{\prime} \mathrm{N} 106^{\circ} 40^{\prime} \mathrm{E}, 600-650 \mathrm{~m}, 28$ Nov 1998, L.A. Averyanov et al. 746 (MO); near Lung
$\mathrm{Mu}$, about $14 \mathrm{~km} \mathrm{~N}$ of Cao Bang, 22 $47^{\prime} \mathrm{N} 106^{\circ} 18^{\prime} \mathrm{E}, 800$ m, 13 Dec 1998, L. Averyanov et al. 1194 (MO); near Thang Heng and Lung Tao, 650 - 1000 m, 25 May 1997, L. Averyanov et al. 4891 (MO). Hà Giang: Bat Dai Son, $27^{\circ} 09^{\prime} \mathrm{N} 105^{\circ} 00^{\prime} \mathrm{E}, 1200$ - $1250 \mathrm{~m}, 04$ Apr 2000, D.K. Harder et al. 5039 (MO); $28^{\circ} 08^{\prime} \mathrm{N} 105^{\circ} 00^{\prime} \mathrm{E}, 1310 \mathrm{~m}, 06$ Apr 2000, D.K. Harder et al. 5238 (MO). Ninh Binh: Cuc Phoung National Park, $600 \mathrm{~m}$, NE of headquarters, $20^{\circ} 14^{\prime} \mathrm{N} 105^{\circ} 43^{\prime} \mathrm{E}, 20$ Dec 1999, N.M. Cuong et al. 768 769 (MO).

\section{Hybrids}

Pistacia $\times$ saportae Burnat, Fl. Alpes Marit. 2:54. 1896. Type: Presumably in Burnat's herbarium at G.

Pistacia lentisco-terebinthus Saporta \& Marion, Ann. Sci. Nat. Bot. ser. 5, 14(1):2. 1871. nom. illeg. (a formula, not a true epithet; ICBN Art. H.10.3).

P. × hybrida Gasp. ex Parl., Fl. Ital. 5:383. 1875. nom. illeg. (a generic name followed by one or more words not intended as a specific epithet; ICBN Art. 23.6.b).

Pistacia lentiscus $\times$ terebinthus Saporta \& Marion ex Engl. in A. \& C. DC., Monogr. Phan. 4:286. 1883. Type: FRANCE: Var: St. Zacharie, Saporta \& Marion (P).

Pistacia saportae var. oxycarpa Zohary, Palestine J. Bot. Jerusalem Ser. 5:204. 1952. TYPE: ITALY: Liguria, near Ventimiglia, 1898, Bickwell (C).

Shrubs, 2 - $3 \mathrm{~m}$ high; branches grayish-brown. Leaves evergreen, imparipinnate, to $10 \mathrm{~cm}$ long coriaceous; rachis narrowly winged. Leaflets $7-9$, to $5.8 \mathrm{~cm}$ long and $2.2 \mathrm{~cm}$ wide, ratio 2.6 - 1, subopposite, sessile, oblong, often falcate, acute, mucronate, glabrous, upper surface darker, margins slightly revolute; terminal leaflet smaller than laterals, linear-lanceolate. Staminate inflorescences not seen. Pistillate inflorescences clustered in racemes or panicles, to $7.5 \mathrm{~cm}$ long. Drupes greenish-pink, globose, 3 - $5 \mathrm{~mm}$ in diameter.

Phenology.-Flowering March to May; fruiting February, September, and October.

Habitat and ecology.-Maquis, calcarious soils; elevation is $450 \mathrm{~m}$.

Distribution.-Scattered through the Mediterranean basin where its supposed parent species, $P$. lentiscus subsp. lentiscus and P. terebinthus, occur. Zohary (1952: 14) discusses several other putative hybrids, but we have seen only those specimens cited below.

Specimens examined. CYPRUS. Akamas Forest, Feb 1955, E. Chapman s.n. (K); between baths of Aphrodite and Fontana Amorosa, 28 Oct 1981, R.D. Meikle 5043 (K). PALESTINE. Upper Galilee, Nabi Yusha, 16 Sep 1951, D. Zohary s.n. (MO); 16 Sep 1951, D. Zohary \& I. Amdursky 440 (BM, MO). SPAIN. Tarragona: Cardó, 450 m, 08 Jun 1946, Font Quer 458 (F). 


\section{Excluded and Problematic Species}

Pistacia americana Mill., Gard. Dict. Ed. 8. N. 7. 1768.

= Simarouba glauca DC., Ann. Mus. Par. 7:424. 1811

(Simaroubaceae)?

Pistacia $\times$ cappadocica Planch., Bull. Soc. Bot. France 11(Sess. Extraord.):46:?. 1864. = Pistacia. terebinthus $\times$ Pistacia. vera?

Pistacia cucphuongensis T.D. Dai, J. Biol. (Vietnam) 3(3):24. 1981. = ? (does not appear to be a Pistacia)

Pistacia fagaroides Willd., Enum, Pl. Suppl. 66. 1814. $=$ ?

Pistacia narbonensis L. Sp. Pl. 2:1025. 1753. pro parte $=$ Pistacia. terebinthus X Pistacia. vera .

Pistacia nemausensis Req. ex Planch., Bull. Soc. Bot. France 11(Sess. Extraord.):46:? 1864. = Pistacia terebinthus $\times$ Pistacia vera.

Pistacia occidentalis Baill., Adansonia 11:181. 1874. $=$ Dacyrodes hexandra Griseb., Fl. Brit. W.I. 174. 1859.

Pistacia oleosa Lour., Fl. Cochinch. 615. 1790. = Schleichera trijuga Willd., Sp. Pl., ed. 4. 4(2):1096. 1806. (Sapindaceae). Zohary (1952), as Schleicheria.

Pistacia simaruba L., Sp. Pl. 2:1026. = Bursera simaruba (L.) Sarg., Gard. \& Forest 3:200. 1890 (Burseraceae).

Pistacia vitex Steud., Nomencl. Bot. 627. 1821. = Vitex pubescens Vahl, Symb. Bot. 3:85. 1794 (Lamiaceae).

\section{Acknowledgements}

This paper results from studies undertaken for a $\mathrm{PhD}$ dissertation at Virginia Polytechnic Institute and State University by AL-Saghir. We thank Brent Opell, Stephen Scheckler, and M.A. Saghai-Maroof for their supervision and valuable comments. We also thank the curators of the Field Museum, Chicago, Illinois (F); Missouri Botanical Garden, St. Louis (MO); Natural History Museum, London (BM); Royal Botanic Garden, Edinburgh (E); Royal Botanic Gardens, Kew (K); and University of Texas, Austin (UT) for the loan of specimens; Thomas Wieboldt (VPI) for arranging this; and Professor Paul Brazhnik for translation of Russian herbarium labels. Charley Jarvis, of the Botany Department, Natural History Museum, kindly commented on typification of Pistacia terebinthus and $P$. vera.

\section{REFERENCES}

[1] P. F. Stevens, "Angiosperm Phylogeny Website, Version 9," 2008.

http://www.mobot.org/MOBOT/research/Apweb/

[2] FAO, "Food and Agriculture Commodities," 2010 http://www.fao.org/es/ess/top/commodity.html

[3] J. Tous and L. Ferguson, "Mediterranean Fruits". In: J. Janick, Ed., Progress in New Crops, ASHS Press, Ar- lington, 1996, pp. 416-430.

[4] D. E. Parfitt and M. L. Badenes, "Phylogeny of the Genus Pistacia as Determined from Analysis of the Chloroplast Genome," Proceedings of the National Academy of Sciences of the United States of America, Vol. 94, No. 15, 1997, pp. 7987-7992. doi:10.1073/pnas.94.15.7987

[5] S. Kafkas and R. Perl-Treves, "Morphological and Molecular Phylogeny of Pistacia Species in Turkey," Theorotical and Appllied Genetics, Vol. 102, 2001, pp. 908915.

[6] M. G. AL-Saghir, "Evolutionary History of the Genus Pistacia (Anacardiaceae)," International Journal of Botany, Vol. 5, No. 3, 2009, pp. 255-257. doi:10.3923/ijb.2009.255.257

[7] M. G. AL-Saghir, "Phylogenetic Analysis of the Genus Pistacia L. (Anacardiaceae) Based on Morphological Data," Asian Journal of Plant Science, Vol. 9, No. 1, 2010, pp. 28-35. doi:10.3923/ajps.2010.28.35

[8] S. Kafkas and R. Perl-Treves, "Inter-Specific Relationships in the Genus Pistacia L. (Anacardiaceae) Based on RAPD Fingerprints," The Journal of Horticultural Science \& Biotechnology, Vol. 37, 2002, pp. 168-171.

[9] S. Kafkas, E. Kafkas and R. Perl-Treves, "Morphological Diversity and a Germplasm Survey of Wild Pistacia Species in Turkey," Genetic Resources and Crop Evolution, Vol. 49, No. 3, 2002, pp. 261-270. doi:10.1023/A:1015563412096

[10] M. Zohary, "A Monographical Study of the Genus Pistacia," Palestine Journal of Botany Jerusalem, Vol. 5, 1952, pp. 187-228.

[11] C. Linnaeus, "Species Plantarum," Laurentii Salvii, Vol. 2, 1753, pp. 1025-1026.

[12] J. P. Tournefort, "Institutiones Rei Herbariae," Typographia Regia, Vol. 2, 1700, pp. 579-580.

[13] F. Yaltirik, “Anacardiaceae,” In: P. H. Davis, Ed., Flora of Turkey and the East Aegean Islands, Edinburgh University Press, Edinburgh, Vol. 2, 1967, pp. 544-548.

[14] F. Yaltirik, "Anacardiaceae, Contributions to the Taxonomy of Woody Plants in Turkey," Notes Royal Botanical Gardan, Vol. 28, 1967, pp.11-12.

[15] IPNI, "The International Plant Name Index," 2010. http://www.ipni.org

[16] T. Yi, J. Wen, A. Golan-Goldhirsh and D. E. Parfitt, "Phylogenetics and Reticulate Evolution in Pistacia (Anacardiaceae)," American Journal of Botany, Vol. 95, No. 2, 2008, pp. 241-251. doi:10.3732/ajb.95.2.241

[17] A. Golan-Goldhirsh, O. Barazani, Z. S. Wang, D. K. Khadka, J. A. Saunders, V. Kostiukovsky and L. J. Rowland, "Genetic Relationships among Mediterra Nean Pistacia Species Evaluated by RAPD and AFLP Markers," Plant Systematics and Evolution, Vol. 246, No. 1-2, 2004, pp. 9-18. doi:10.1007/s00606-004-0132-4

[18] S. Kafkas, "Phylogenetic Analysis of the Genus Pistacia by AFLP Markers," Plant Systematics and Evolution, Vol. 262, No. 1-2, 2006, pp. 113-124. doi:10.1007/s00606-006-0460-7

[19] M. AL-Saghir and D. M. Porter, "Random Amplified 
Polymorphic DNA (RAPD) Study of Pistacia Species (Anacardiaceae)," Asian Journal of Plant Science, Vol. 5, No. 6, 2006, pp. 1002-1006. doi:10.3923/ajps.2006.1002.1006

[20] A. Engler, "Syllabus der Pflanzenfamilien," Gebrüder Borntraeger, Berlin, Vol. 11, 1936.

[21] D. L. Swofford, "PAUP: Phlyogenetic Analysis Using Parsimony 4.0 b," Sinauer Associates, Inc., Sunderland, 2003.

[22] J. McNeil, F. R. Barrie, H. M. Burder, V. Demou, D. L. Hawksworth, K. Masrhold, D. H. Nicolson, J. Prado, P. C. Silva, J. E. Skig, J. H. Wiersema and N. J. Turland, "International Code of Botanical Code (Vienna Code)," Regnum Vegetable, Vol. 146, 2006.

[23] GRIN-CA, "Taxonomy," 2010.

http://pgrc3.agr.gc.ca/cgi-bin/npgs/html/econ.pl
[24] W. T. Stearn, "An Introduction to the Species Plantarum and Cognate Botanical Works of Carl Linnaeus," Carl Linnaeus Species Plantarum, Vol. 1, 1957, pp. 1-176.

[25] C. Jarvis, "Order Out of Chaos. Linnean Plant Names and Their Types," The Linnean Society, London, 2007.

[26] H. O. Juel, “Joachim Burser's Hortus Siccus Mit Erklärungen Herausgegeben," Symbolae Botanicae Upsalienses, Vol. 2, No. 1, 1936. pp. 1-187.

[27] S. Savage, "Catalogue of the Manuscripts in the Library of the Linnean Society of London. Part II. Caroli Linnaei Determinationes in Hortum Siccum Joachimi Burseri," The Linnean Society, London, 1937.

[28] C. Bauhin, "Pinax Theatri Botanici," 2nd Edition, Ludovici Regis, Basel, 1671. 